1 Environmental and physiological factors affecting high-throughput measurements of

\section{bacterial growth}

Esha Atolia ${ }^{1}$, Spencer Cesar ${ }^{2}$, Heidi A. Arjes ${ }^{3}$, Manohary Rajendram $^{3}$, Handuo Shi²,3, Benjamin D. Knapp ${ }^{4}$, Somya Khare³, Andrés Aranda-Díaz ${ }^{3}$, Richard E. Lenski ${ }^{5,6}$, Kerwyn Casey Huang $2,3,4,7$ * $^{*}$

${ }^{1}$ Department of Chemical and Systems Biology, Stanford University School of Medicine, Stanford, CA 94305, USA

${ }^{2}$ Department of Microbiology and Immunology, Stanford University School of Medicine, Stanford, CA 94305, USA

${ }^{3}$ Department of Bioengineering, Stanford University, Stanford, CA 94305, USA

${ }^{4}$ Biophysics Program, Stanford University School of Medicine, Stanford, CA 94305 USA

${ }^{5}$ Department of Microbiology and Molecular Genetics, Michigan State University, East Lansing, MI 48824 USA

${ }^{6}$ BEACON Center for the Study of Evolution in Action, Michigan State University, East Lansing, MI 48824 USA

${ }^{5}$ Chan Zuckerberg Biohub, San Francisco, CA 94158 
21 Abstract

Bacterial growth in nutrient-rich and starvation conditions is intrinsically tied to the environmental history and physiological state of the population. While highthroughput technologies have enabled rapid analyses of mutant libraries, technical and biological challenges complicate data collection and interpretation. Here, we present a framework for the execution and analysis of growth measurements with improved accuracy over standard approaches. Using this framework, we demonstrate key biological insights that emerge from consideration of culturing conditions and history. We determined that quantification of the background absorbance in each well of a multi-well plate is critical for accurate measurements of maximal growth rate. Using mathematical modeling, we demonstrated that maximal growth rate is dependent on initial cell density, which distorts comparisons across strains with variable lag properties. We established a multiple-passage protocol that alleviates the substantial effects of glycerol on growth in carbon-poor media, and we tracked growth ratemediated fitness increases observed during a long-term evolution of Escherichia coli in low glucose concentrations. Finally, we showed that growth of Bacillus subtilis in the presence of glycerol induces a long lag in the next passage due to inhibition of a large fraction of the population. Transposon mutagenesis linked this phenotype to the incorporation of glycerol into lipoteichoic acids, revealing a new role for these envelope components in resuming growth after starvation. Together, our investigations 
bioRxiv preprint doi: https://doi.org/10.1101/2020.06.16.156182; this version posted June 18, 2020. The copyright holder for this preprint (which was not certified by peer review) is the author/funder. All rights reserved. No reuse allowed without permission.

41 underscore the complex physiology of bacteria during bulk passaging and the 42 importance of robust strategies to understand and quantify growth. 


\section{Abstract Importance}

How starved bacteria adapt to and multiply in replete nutrient conditions is intimately linked to their history of previous growth, their physiological state, and the surrounding environment. While automated equipment has enabled high-throughput growth measurements, data interpretation and knowledge gaps regarding the determinants of growth kinetics complicate comparisons between strains. Here, we present a framework for growth measurements that improves accuracy and attenuates the effects of growth history. We determined that background absorbance quantification and multiple passaging cycles allows for accurate growth-rate measurements even in carbon-poor media, which we used to reveal growth-rate

54 increases during long-term laboratory evolution of Escherichia coli. Using mathematical modeling, we showed that maximum growth rate depends on initial cell density. growth of most of the population, due to lipoteichoic-acid synthesis. These studies highlight the challenges of accurate quantification of bacterial growth behaviors. 


\section{Introduction}

Precise growth measurements are fundamental to our understanding of bacterial physiology and its regulation. While some bacterial species are among the fastestgrowing organisms on the planet, others grow imperceptibly slowly, with doubling times ranging from $\sim 7$ minutes [1] to thousands of years [2]. Although the need for rapid growth may drive selection in some cases, many bacteria live in complex natural environments that are often stressful and nutrient limited [3]. In many environments, such as the mammalian gut, leaf litter in soil, and whale falls in the ocean, food is provided only periodically, and hence bacteria experience cycles of feast and famine. Thus, the transition from starvation to rapid growth can also act as an important selective pressure in evolution [4-6]. initial overnight growth of a liquid culture, from either a frozen stock or a colony, which is then used to inoculate fresh medium for optical-density (OD) measurements in a plate reader or spectrophotometer over time [7] (Fig. 1A). Although there are exceptions when OD does not track viable cell number [8,9], OD is widely used as a

76 proxy for the density of cells in a culture $[10,11]$. While traversing such a growth curve,

7 a cell population initially takes some time to accelerate in growth, experiences a period of rapid growth, and then decelerates as nutrients are consumed, waste products 
accumulate, or both [7]. While there can be substantial variability in the shape of the growth curve, many species qualitatively exhibit a sigmoidal shape that can be characterized by three parameters: (1) the maximal growth rate, $\mu_{\max }$, which is the largest slope of the natural log of the OD over time, (2) the lag time required to accelerate growth from stationary phase $\left(T_{\mathrm{lag}}\right)$, and (3) the maximum or final OD $\left(A_{\max }\right)$; some cultures also exhibit a death phase [7]. These parameters are typically extracted from a growth curve via fitting or direct calculation [12-21]. Long-term evolution experiments (LTEEs) have demonstrated that all three can be under selection [4, 22], underscoring the importance of their accurate quantification. However, we have little understanding of how the technical aspects of model fitting and the methodological aspects of inoculation affect such quantifications.

The creation of genome-wide knockout libraries $[23,24]$ has motivated high-throughput measurements of population growth - a commonly used proxy for fitness-in microtiter plates, such as the systems-biological characterization of essential gene knockdowns in Bacillus subtilis [5] and of Escherichia coli non-essential gene knockouts in liquid $[25,26]$ or embedded in a gel $[26,27]$. As the development of advanced genetic tools simplifies the creation of strain libraries [28-30], it is critical to ensure that OD measurements in multi-well plates provide reliable estimates of population-level growth parameters. 
100 Here, we provide a detailed analysis of the requirements for achieving robust, high-

101 throughput measurements of fitness parameters. We quantified the importance of

102

103

104

105

106

107

108

109

110

111

112

113

114

115

116

117

118 accurate background subtraction and established an assay of the sensitivity of the plate reader. We determined that measurements of maximal population growth rate and lag time are both sensitive to initial inoculation density, and we developed a mathematical model based on ordinary differential equations that accurately predicts this density dependence. We showed that completely removing residual glycerol from frozen stocks as a carbon source is critical for accurate measurements of E. coli growth in carbonlimited media, and we used such a strategy to reveal that evolution in a carbon-poor medium led to significant increases in the maximal growth rate of a population. We also established that the presence of glycerol during initial outgrowth from frozen stocks can have long-term impact on B. subtilis growth, causing long apparent lag times during subsequent culturing due to growth inhibition in a large majority of cells. Using transposon mutagenesis, we discovered that the increased lag time at least partially results from incorporation of glycerol during lipoteichoic acid synthesis. For large-scale, high-throughput experiments, inoculating a large number of cultures from colonies is often too cumbersome, and thus these considerations are vital. Together, these findings provide a framework for accurate quantification of growth parameters and a roadmap for identifying and controlling for physiological factors that impact growth. 


\section{Results}

To monitor maximum growth rate and lag time (defined here as time to reach halfmaximum growth rate) during a bacterial population's exit from stationary phase, it is standard practice to dilute a stationary-phase culture sufficiently that the spent medium transferred with the cells is a small fraction of the solution relative to the fresh medium. many species in high-nutrient conditions measured with our plate reader), the starting OD is $\sim 0.001-0.01$. Thus, for species such as E. coli for which the maximum growth rate is achieved within 2-3 generations [31], the corresponding OD at the time of maximum growth rate can be low $(\lessgtr 0.01)$ (Fig. $1 \mathrm{~A})$, making it critical to ascertain whether OD can be accurately measured at low cell densities.

134 It is generally appreciated that correcting for the background absorbance improves the

135 accuracy of growth measurements. However, the extent to which different background correction methods affect growth rate calculations has not been quantified. For a culture that is growing exponentially, the number of cells, $N$, grows over time as $N(t)=N_{0} 2^{t / \tau}$, 
138

139 cells; $\mathrm{OD}_{\mathrm{bg}}$ is typically $\sim 0.1$ (Fig. $1 \mathrm{~A}$ ). Without subtraction of $\mathrm{OD}_{\mathrm{bg}}$, the computed growth 156 rate would be $\frac{1}{\alpha N(t)+0 D_{\mathrm{bg}}} \frac{\alpha N(t+\Delta t)-\alpha N(t)}{\Delta t}$ rather than $\frac{1}{\alpha N(t)} \frac{\alpha N(t+\Delta t)-\alpha N(t)}{\Delta t}$; the estimate of

where $N_{0}$ is the number of cells at $t=0$ and $\tau$ is the doubling time. Thus, the growth rate of such a culture can be defined as the constant

$$
\frac{1}{N(t)} \frac{d N(t)}{d t}=\frac{\ln 2}{\tau}
$$

During outgrowth from stationary phase, when cells adapt their proteome to exploit the newly available nutrients [32], or after the cell density is sufficiently high that growth modifies the environment in a manner that impacts cellular physiology, the population does not grow exponentially. Nonetheless, analogous to exponential growth, we can define an instantaneous growth rate as

$$
g(t)=\frac{1}{N(t)} \frac{d N(t)}{d t}=\frac{d(\ln N(t))}{d t}
$$

Assuming that the OD measured by a plate reader is linearly related to $N(\mathrm{OD}=\alpha N$, where $\alpha$ is a scaling factor relating cell number to OD) and measurements are taken at time points $t, t+\Delta t, t+2 \Delta t$, etc., the instantaneous growth rate at time $t$ can be estimated as

$$
g(t)=\frac{1}{\mathrm{OD}(t)} \frac{d(\mathrm{OD}(t))}{d t} \approx \frac{1}{\mathrm{OD}(t)} \frac{\mathrm{OD}(t+\Delta t)-\mathrm{OD}(t)}{\Delta t}
$$

To illustrate the importance of background subtraction, consider a culture in which $\mathrm{OD}_{\text {raw }}(t)=\alpha N(t)+\mathrm{OD}_{\mathrm{bg}}$, where $\mathrm{OD}_{\mathrm{bg}}$ is the background absorbance in the absence of 
157 growth rate would be incorrect by a factor of $\frac{\alpha N(t)}{\alpha N(t)+0 \mathrm{D}_{\mathrm{bg}}}$ (Fig. 1A). Thus, any estimate of growth rate without background (blank) subtraction is highly underestimated when in the regime $\mathrm{OD}_{\mathrm{bg}} \gtrsim \alpha N(t)$, which is likely for the time at which the maximum growth rate of many bacterial species is first achieved. For similar reasons, using the first time

161 point of a growth curve as a proxy for the background absorbance leads to overestimation of the maximum growth rate because the subtracted background was too large. Therefore, a method for correctly subtracting the background is crucial for accurate growth-rate estimates.

We measured growth curves of E. coli MG1655 and estimated the instantaneous growth rate over time (Methods), with and without blank subtraction. After subtracting the well blank, the maximal growth rate was $1.83 \mathrm{~h}^{-1}$ (doubling time of $22.7 \mathrm{~min}$ ), which occurred at $t=1.52 \mathrm{~h}$ (Fig. 1B). Without blank subtraction, the maximal growth rate estimate was substantially lower $\left(0.49 \mathrm{~h}^{-1}\right)$ and the time at which this inaccurate estimate occurred was $t=3.0 \mathrm{~h}$ (Fig. 1B), illustrating the effects of omitting blank subtraction on

172 lag time. To determine whether one empty well could be used as a general proxy for background absorbance, we quantified the absorbance of each well with medium before

174 inoculating cells (Methods). Blank values varied by $\sim 0.004$ across the plate, while a 175 single well's blank value fluctuated by $<0.001$ over time (Fig. 1C). Blanking with a 176 randomly selected well from the plate led to a wide variation in blanked growth curves 
177 (Fig. 1D) and maximum growth rates (Fig. 1E) with a standard deviation in growth-rate

178 estimate of $0.52 \mathrm{~h}^{-1}$ (Fig. 1F). Background subtraction with a well-specific blank led to

179 substantially less variation in growth-rate measurements, with a standard deviation in

180 growth-rate estimates for replicate cultures across the plate of $0.15 \mathrm{~h}^{-1}$ (Fig. 1F). Well-

181 specific blanking also decreased the variability in lag measurements (Fig. S1A). The

182 within-plate variability is sufficient to change the rank ordering of growth rates, which

183 can lead to erroneous inferences. Thus, well-specific blanking is critical for accurate

184 measurements of growth rate, because even comparisons in a single plate are

185 confounded by within-plate variability.

186

Sensitivity limit of the spectrophotometer also impacts the accuracy of growth-rate

188

189

190

191

192

193

194

195

196

\section{measurements}

The ability to accurately measure changes in OD across a range of cell densities spanning several orders of magnitude is equally important for growth-rate calculations.

Thus, we sought a general protocol for quantifying the limit of sensitivity and linear range of a given spectrophotometer. We inoculated serial dilutions of an overnight culture of E. coli MG1655 into fresh LB and measured the OD values. At low dilutions

$(\mathrm{OD}>0.63)$, the absorbance measured by the plate reader was not linearly related to cell density (Fig. 1G,H). Nonetheless, high OD measurements could be converted to celldensity estimates with a measured calibration curve (Fig. 1G), because the measurement 
coefficient of variation (CV; standard deviation/mean) remained very low (Fig. 1I). To extended up to the maximum tested expected OD of $\sim 3.5$ (Fig. 1G), which is substantially higher than the typical corrected OD of $\sim 1$ for a stationary phase E. coli culture grown in LB. to the dilution factor (Fig. 1G) and had a CV $<0.2$ (Fig. 1I) down to a blank-corrected OD of $\sim 0.006$, well below the plate background $\mathrm{OD}_{\mathrm{bg}}$. For larger dilutions $(\mathrm{OD}<0.006)$, the spectrophotometer are likely to be very noisy for $\mathrm{OD}<0.006$. We conclude that any growth curve should be initialized with an inoculum such that the maximum growth

211 rate is achieved above a blank-corrected value of 0.006 . This strategy is likely effective

212 for calibrating and determining the sensitivity limit of any spectrophotometer.

215 We previously found that the instantaneous population growth rate was strongly 216 correlated with OD across a library of B. subtilis mutants, despite their widely varying 
217 lag times, suggesting that cell density plays a major role in determining the 218 population's growth rate [5]. Thus, to determine the optimal dilution for initializing 219 growth curves, we systematically quantified how the inoculum cell density affects the trajectory of outgrowth from stationary phase. We diluted an overnight culture of E. coli MG1655 into fresh LB at ratios ranging from 1:12.5 to 1:6400 in a 96-well plate, and monitored the growth curves (Fig. 2A). To examine the relationship between OD and growth rate, we plotted each curve as growth rate versus $\log _{10}(\mathrm{OD})$ (Fig. 2B). After correcting for the nonlinearity at high ODs (Fig. 1G), and subtracting the well-specific backgrounds, we found that the maximum growth rate achieved at low dilutions (e.g., 1:12.5) was lower than at larger dilutions (Fig. 2B). As expected, each curve started at a different initial OD with a growth rate near 0 (Fig. 2B). Growth rate then increased, and 228 for large dilutions (1:6400) reached $\mu_{\max } \approx 2 \mathrm{~h}^{-1}$ (Fig. 2B). However, for lower dilutions, $\mu_{\max }$ was substantially less than $2 \mathrm{~h}^{-1}$ and was attained at higher OD (Fig. 2B). In each case, after reaching $\mu_{\max }$ growth rate declined approximately linearly as a function of $231 \log _{10}(\mathrm{OD})$ along a common trajectory (Fig. 2B). Thus, before a population reaches its 232 maximum growth rate, its growth rate trajectory is dependent on the initial cell density; 233 thereafter, the growth curve follows a prescribed path independent of initial cell 234 density. 
236 To interrogate whether factors such as nutrient depletion or waste accumulation cause

237 this density dependence, we developed a minimal model of population growth

238 dynamics during passage in liquid culture. We assume that cell density $C$ grows with

239 an instantaneous growth rate $\mu$ dictated by the physiological status of the cells and the

240 external environment:

$$
\frac{d C}{d t}=\mu C
$$

242 Nutrients are consumed by growing cells at a rate proportional to their growth rate:

$$
\frac{d n}{d t}=\omega C-\beta \mu C
$$

244 where $n$ is nutrient concentration, $\omega$ represents production of nutrients by the cells, and

$245 \beta$ is the nutrient consumption rate. We assume that growth rate is a function of the 246 nutrient concentration relative to a fixed nutrient concentration $K$ [7]; to model the 247 transition from stationary phase into $\log$ phase, we assume that $\mu$ is related to the 248 highest possible maximal growth rate $\mu^{*}$ via a Gompertz relation [33]:

$$
\mu=\mu^{*}\left(\rho_{\max } e^{-e^{\frac{\lambda e(\delta-t)}{\rho_{\max }}+1}}+\rho_{\min }\right) \frac{n}{n+K},(6)
$$

250 where $\rho_{\min }$ is the lowest growth rate attained at high nutrient concentration normalized

251 to $\mu^{*}, \rho_{\max }=1-\rho_{\min }, \lambda$ governs how quickly $\mu$ increases, and $\delta$ is the time required to 252 reach the maximum rate of growth-rate change. We used single-cell growth data to 253 obtain estimates of $\rho_{\max }, \rho_{\min }, \lambda$, and $\delta$ (Fig. S1B). We found that the simulated growth 
254 curves were relatively insensitive to the precise functional form of the acceleration in growth during lag phase.

We simulated growth curves based on Eqs. (4-6), assuming that OD is proportional to $C$, with different initial densities $C(t=0)$ and $K=0.5$ (where $n=1$ is the maximum nutrient level), $\mu^{*}=2 \mathrm{~h}^{-1}, \beta=0.8 \mathrm{~h}, \rho_{\max }=0.99, \rho_{\min }=0.01, \lambda=0.8 \mathrm{~h}^{-1}$, and $\delta=0.5 \mathrm{~h}$. The kinetics of these growth curves (Fig. 2C) and the resulting relation between growth rate and OD (Fig. 2D) recapitulated our experimental findings reasonably well (Fig. 2B), including the roughly linear decrease in growth rate with OD after reaching $\mu_{\max }$. Hence, nutrient depletion can largely explain the relations between $\mu_{\max }$ and

264 inoculation density (Fig. 2E), between lag and inoculation density (Fig. S1C), and more 265 generally between growth rate and OD (Fig. 2D). To illustrate the importance of these results, we examined the growth of a library of $E$.

268 coli cell-size mutants [34]. After a 1:10,000 dilution, all mutants exhibited similar maximum growth rates (Fig. 2F). However, after a 1:200 dilution, maximum growth rate was negatively correlated with the average cell width of each mutant (Fig. 2F) [34]. This effect appears to reflect differences in outgrowth that prevented many of the mutants

272 from attaining the higher maximum growth rate achievable at lower inoculation 
274 low a cell density as possible without dropping below the plate reader's limit of

275 detection, in order to avoid the region of decreasing maximum growth rate at high cell

276 densities (Fig. 2F) that can distort comparisons between strains.

\section{Growth in a carbon-poor medium is highly sensitive to glycerol levels}

279 High-throughput methods often involve inoculation directly from a frozen stock rather

284 times. We hypothesized that glycerol would have persistent effects on growth,

285 particularly in nutrient-poor media, because it can be utilized as a carbon source.

286 Glycerol use that substantially increases the number of cells during the first passage evolution experiments, which are often carried out in media with low carbon

290 concentrations [37]. To test the effect of glycerol on growth, we measured growth 291 curves across a range of glycerol concentrations (Fig. 3A-D). We inoculated $1 \mu \mathrm{L}$ from a $292-80{ }^{\circ} \mathrm{C}$ freezer stock (previously grown in Davis Minimal medium with $25 \mathrm{~g} / \mathrm{L}$ glucose, 
294 evolution experiment (LTEE) carried out by Lenski and colleagues, into the evolution 295 medium (DM25) supplemented with 0-10\% (v/v) glycerol (Fig. 3A,B). The addition of 296 glycerol mimics various levels of glycerol carryover from frozen stocks during 297 inoculation. (However, we emphasize that the growth rate and competitive fitness 298 assays performed by Lenski and collaborators prevent carryover of glycerol through 299 repeated culturing in DM25, which acclimates the bacteria to the medium and other 300 conditions of the LTEE prior to the fitness assays [39]). When we diluted these cultures 301 1:200, the resulting initial ODs substantially differed across glycerol concentrations, 302 resulting in different growth kinetics with higher final OD values for cultures coming 303 from higher glycerol concentrations on day 2 (Fig. 3C,E). After passaging the cultures a 304 third time, the growth curves for the various glycerol concentrations were now 305 quantitatively similar (Fig. 3D) with lower final ODs, as expected (Fig. 3E). Similar 306 glycerol-dependent effects appeared when different amounts of frozen stock were 307 inoculated into DM25 (Fig. S2A,B). Thus, accurate quantification of growth requires 308 multiple passages to eliminate the effects of glycerol on growth.

310 Combined with our finding that growth rate can be accurately measured even at low 311 OD values with well-specific background subtraction (Fig. 1F), we realized that we 312 could use multiple passaging to measure growth parameters in a high-throughput 313 manner for the LTEE strains, enabling us to determine whether they changed 
314 systematically over the course of the LTEE. We examined 12 strains sampled from the

315 Ara-1 population through 60,000 generations [40,41]. We diluted each culture into fresh

316 DM25, and measured their growth curves. We then re-diluted these overnight cultures strains attained relatively high ODs in the first passage due to the residual glycerol (Fig. $\mathrm{S} 2 \mathrm{E})$. In the second passage, growth rates were higher for the strains from later generations, but the measurements were noisy due to variations in inoculum levels (Fig. the fourth passage, maximum growth rates of the evolved strains had converged on

324 values close to those measured the previous day (Fig. 3G), ranging from $\sim 0.65 \mathrm{~h}^{-1}$ to

$325 \sim 1.08 \mathrm{~h}^{-1}$ from the earliest to the latest sample. Notably, the low stationary-phase density in DM25 (Fig. S2E) meant that even small amounts of noise greatly affected the measurements of growth rate; hence, well-specific blanking instead of random-well blanking was critical (Fig. 3E-G). 
suitable for measuring growth rates. Our new measurements highlight the importance of accurate blanking for quantifying growth behaviors, especially at low OD values. 4Bi, S3Ai) and similar lag times (Fig. 4Bii, S3Aii).

349 cells as they progress through another passage. To determine whether subsequent

350 growth was affected by the prior presence of glycerol, we diluted all cultures 1:200 into 
354 This increased lag was specific to B. subtilis (Fig. S3Bii, S3Eii, S3Hii), and it went away

355 during a third passage (Fig. 4D). Similar behavior was seen when inoculating a 96-well

356 plate with fresh LB with various volumes $(0.1-100 \mu \mathrm{L})$ of thawed frozen stocks of $B$.

357 subtilis 168 (Fig. 4A, S3D-F). These data indicate that the second passage after revival of

358 B. subtilis from a frozen stock is very sensitive to the glycerol concentration during

359 initial inoculation.

361 To confirm that cellular responses to freezing were not required for the increase in lag

362 time, we used $1 \mu \mathrm{L}$ of an overnight culture grown from frozen stock to inoculate LB

363 supplemented with $0.1 \%$ to $10 \%$ glycerol; these cultures were now removed from

364 freezing by a 24-h passage (Fig. S4A, purple). During the passage after glycerol

365 addition, cells exhibited the expected increases in lag time (Fig. S4A, purple).

366 Furthermore, when we inoculated the initial culture from a colony (instead of a frozen

367 stock) into LB supplemented with glycerol, we saw similar increases in lag time on the

368 subsequent passage (Fig. S4A, yellow) as from inoculating in varying amounts of

369 glycerol (Fig. 4C; S4A, teal) or different amounts of frozen stock (Fig. S3E). These

370 findings suggest that regrowth from frozen stock displays little to no sign of cell death

371 relative to regrowth from a colony. The long-lag phenotype in the presence of glycerol

372 for B. subtilis is distinct from the effects we observed in E. coli (Fig. 3B-E), in which the

373 glycerol was metabolized and thus led to higher carrying capacities. Altogether, these 

cells. Such extreme bottlenecks should be avoided for most applications.

data show that growth of $B$. subtilis 168 in glycerol can cause dramatic lag time increases during the subsequent passage for intermediate concentrations of glycerol. They highlight the importance of controlling for glycerol levels during high-throughput growth assays, which can be readily achieved by performing an additional passage.

\section{Glycerol has dramatic and varied effects on B. subtilis single-cell growth}

We were surprised by the increased lag times in B. subtilis growth after passaging with intermediate glycerol concentrations, and sought to investigate the cell physiology underlying this phenomenon. We used time-lapse microscopy to monitor the growth of cells on LB agarose pads following growth in liquid LB at the glycerol concentrations associated with the longest population lag times (Fig. 4C). For cells grown in liquid LB with $0.3125 \%$ glycerol ( $\sim 6 \mathrm{~h}$ lag time, Fig. 4Cii), we imaged an entire $\sim 2 \mathrm{~mm}$-diameter spot by capturing a grid of 144 overlapping fields of view. Of the $\sim 10^{4}$ cells in one spot, only a single cell grew, and its descendants took over the entire spot over $12 \mathrm{~h}$ of imaging (Fig. 5B). That cell exhibited growth as soon as imaging began, and after $1.7 \mathrm{~h}$ its lineage exhibited a doubling time of $\sim 20 \mathrm{~min}$ (Fig. 5C), indicating the extreme heterogeneity in this population. In other spots $(n=2)$, we observed no growth of any 
393 For cells grown in LB with $0.625 \%$ glycerol ( $\sim \mathrm{h}$ lag time, Fig. 4Cii), we again observed

394 a small fraction of growing cells $(<3 \%)$ (Fig. 5D). The cells that grew showed multiple

\section{A screen links glycerol-induced lag to genes involved in teichoic acid synthesis}

406 The discovery of $B$. subtilis's long lag and consequent fitness defect induced by

407 intermediate glycerol concentrations (Fig. 4) presented the opportunity to identify

408 genetic determinants of this phenotype, as mutants with a shorter lag would be 409 enriched in the population. To gain insight into the underlying mechanism, we carried

410 out an unbiased genetic screen by creating six independent pooled libraries of 411 transposon mutants (Methods) [44] in the wild-type strain. We grew the libraries in LB $412+1.25 \%$ glycerol, a concentration that induced a long lag time in wildtype (Fig. 5Eii). 
413 Further passaging of the libraries once in LB $+1.25 \%$ glycerol and once more in LB

414 revealed two libraries that evolved shorter lag times (Fig. S5B). We isolated single

415 colonies and verified that they had a similar phenotype to the library from which they

416 were isolated (Fig. S5C). Sequencing their transposon insertion sites identified two

417 mutations: in sigX, which encodes a sigma factor that regulates modification of the cell

418 envelope and resistance to cationic antimicrobial peptides [45], and in the start codon of

$419 \operatorname{dlt} A$, which encodes a D-alanine ligase required for modification of wall teichoic acids

420 (WTA) and lipoteichoic acids (LTA) [46]. Note that dltA is part of the sigX regulon (Fig.

421 5G) [45]. We verified these hits by reintroducing each transposon insertion into the

422 parental strain (Fig. S5D,E) and by deleting the gene ( $\operatorname{sig} X$ or $\operatorname{dlt} A)$, and then showing

423 that these constructs exhibited the same reduction in lag (Fig. 5H,I). The thick

424 peptidoglycan cell wall of Gram-positive bacteria is intercalated with wall teichoic

425 acids, which are covalently bound to peptidoglycan, and lipoteichoic acids, which are

426 tethered to the membrane by a lipid anchor [47]. Production of both wall teichoic acids

427 and lipoteichoic acids requires substantial amounts of glycerol [48]. Thus, it appears

428 that teichoic-acid production is linked to the glycerol-dependent long-lag phenotype in

429 B. subtilis. 


\section{Discussion}

431 As microbiology research has expanded and flourished, so has the appreciation of the 432 sensitivity of microbial physiology and cellular structure to environmental conditions.

433 Uncovering the details of these sensitivities will be critical to quantitative 434 understanding of growth behaviors across microbial strains and species, as will 435 establishing the resolution and robustness of the equipment used to measure growth. 436 We have described a general strategy for measuring an instrument's limit of detection 437 and range of linearity, and demonstrated that, with proper protocols, a wide range of growth behaviors can be accurately quantified.

440 The dependence of maximum growth rate on initial cell density presents complications 441 similar to the antibiotic inoculum effect, whereby the sensitivity to certain drugs 442 increases at lower cell density [49]. Comparisons of growth rate and lag time between strains would ideally employ similar initial cell concentrations. However, fulfilling such

444 a condition can be challenging due to strain differences in cell shape [50], yield in a 445 given medium, and cell survival in and recovery from stationary phase. Moreover, 446 some species may have growth dynamics and carrying capacities that are inoculum447 dependent. Given these complications, the acquisition of growth curves across a range 448 of initial densities to map the range of growth behaviors would enhance the ability to compare strains and species. Our model based on ordinary differential equations is 
450 general and hence can be used for many microbes; in particular, it can help to correct

451 for differences in growth rate due to variation in initial inoculum size. It is also

452 important to note that waste accumulation should be mathematically equivalent to

453 nutrient depletion if waste products inhibit the uptake of some nutrients, which means

454 our model is even more broadly applicable. However, other factors may prove

455 important for modeling growth curves, such as $\mathrm{pH}$ changes that are known to inhibit

456 growth [51].

457

Although spectrophotometers that read microtiter plates are quite sensitive to small changes in OD (Fig. 1D,E), our analyses establish that it is critical to minimize noise that introduces complexities when calculating growth metrics; this noise minimization can

461 be best achieved by blanking each individual well separately. This modification to 462 protocols was relatively straightforward, and it positioned us to quantify the contribution of increased growth rate to the fitness gains observed in the LTEE with $E$.

464 coli (Fig. 3). In particular, this method allowed us to show unequivocally that faster 465 exponential growth had been selected even at the low glucose concentration and 466 consequently low OD values of the LTEE. The $\sim 66 \%$ increase in maximum growth rate 467 that we measured is reasonably close to the $\sim 70 \%$ increase in relative fitness obtained 468 through competing late-generation samples against their ancestor [39]; that relative 
470

471

472

473

474

475

476

477

478

479

480

481

482

483

484

485

486

487

488

489 bacteria over a full 24-hour transfer cycle including lag, growth, and stationary phases.

Thus, other growth parameters can also affect relative fitness including differences in

lag time and carrying capacity [52], which complicates a direct comparison between

maximal growth rate and relative fitness. In addition, crossfeeding interactions have

evolved in some LTEE populations, and these interactions may affect the post-

maximum growth rates of the competitors as cells exhaust the limiting glucose,

consume byproducts, and transition into stationary phase [53-55]. In any case, our new

protocol and growth-rate measurements demonstrate the value of subtracting the blank

of each well to minimize noise, especially at low OD values. These growth-rate data also

imply that utilization of glucose has become much faster over the LTEE, and more

generally it may be possible to evolve many bacterial species to grow at higher rates in

specific nutrient conditions. Given the correlation between cell size and fitness in the

LTEE [56], future studies might use these strains to explore whether the "Growth Law"

that links nutrient-dependent growth rate and cell size [57] has changed over the course

of evolution. In fact, it was previously shown that that the relation is not constant

between the ancestor and an evolved strain isolated after just 2,000 generations [58].

The dramatic increases in lag time that we observed in B. subtilis (Fig. 4C) indicate that

glycerol can have lasting physiological effects that impede the future growth of cells.

We did not observe this lag phenotype in E. coli (Fig. S3B). This difference is consistent 
with the requirement of Gram-positive bacteria for glycerol to synthesize teichoic acids,

491 which they incorporate into the cell envelope. Without multiple dilutions to mitigate

492 the glycerol-induced long-lag phenotype, a severe bottleneck in which very few cells are responsible for outgrowth can occur (Fig. 5B), which may complicate interpretation.

494 The conventional approach to streaking single colonies before starting liquid cultures 495 avoids this problem (Fig. S4A,B), likely due to the extreme dilution of the glycerol concentration. While streaking may be prohibitive for large strain collections or certain species and communities, washing the initial inoculum to remove the glycerol is also sufficient (Fig. S2C,D).

Our transposon mutagenesis screen linked the glycerol-induced long-lag phenotype in the subsequent passage including filamentation (Fig. 5D), bulging (Fig. 5E), lysis (Fig. S5A), and repeated cycles of growth and shrinkage (Fig. S5A). Some of these phenotypes are consistent with previous observations connecting lipoteichoic-acid

506 synthesis with cell division [59]. Moreover, the arrest of growth in the vast majority of 507 cells (Fig. 5E) indicates that their physiological state was sensitized by previous exposure to even low amounts of glycerol, such that growth remained inhibited even 
510 to growth inhibition and lag phase for the first time, and it points to a severe bottleneck

511 that might cause additional experimental complications. This surprising phenotype also

512 highlights the potential for other physiological history-dependent effects on growth.

514 In the process of dissecting the seemingly simple process of measuring bacterial growth,

515 we developed a refined protocol that enabled precise, quantitative measurements. This

516 precision, in turn, led to the discovery of new biological phenomena. The microbial

517 world is stunningly diverse, and we currently know very little about the growth

518 kinetics of the vast majority of microbes. Our work provides a powerful framework to

519 analyze the growth characteristics of microbial species in high-throughput assays. 


\section{Methods and Materials}

\section{Strains and media}

522 Table S1 lists the strains and their genotypes used in this study. Strains were grown in

524 (Davis minimal broth [60] supplemented with $2 \times 10^{-3} \mathrm{~g} / \mathrm{L}$ thiamine hydrochloride and

$52525 \mathrm{mg} / \mathrm{L}$ glucose [37]). Both media were supplemented with 0-10 \% (v/v) glycerol when

526 indicated. When stated, antibiotics were used as follows, unless indicated otherwise:

527 kanamycin $(5 \mu \mathrm{g} / \mathrm{mL}$ ) and MLS (a combination of $0.5 \mu \mathrm{g} / \mathrm{mL}$ erythromycin and 12.5

$528 \mu \mathrm{g} / \mathrm{mL}$ lincomycin).

Bacterial growth

531 For all glycerol experiments, to avoid potential issues associated with accumulated

532 damage over time spent in the freezer, we first made new frozen stocks of E. coli

534 freezing a 1:1 mixture of the culture with $50 \%$ glycerol in a $-80{ }^{\circ} \mathrm{C}$ freezer. All strains

535 (Table S1) were inoculated from $-80{ }^{\circ} \mathrm{C}$ freezer stocks into $200 \mu \mathrm{L}$ of the medium of

536 interest in shallow 96-well plates (Greiner) and grown overnight for $18 \mathrm{~h}$ while shaking

537 at $37^{\circ} \mathrm{C}$. Overnight cultures were diluted 1:200 into fresh medium for plate-reader 538 experiments, and either 1:10 or 1:20 into fresh medium for microscopy. 
541 A shallow 96-well plate was filled with fresh medium, and a plate seal (Excel Scientific)

542 with laser-cut holes was placed on top. The background absorbance at $600 \mathrm{~nm}\left(\mathrm{OD}_{600}\right)$ was measured for $30 \mathrm{~min}$ to allow the readings to stabilize. Overnight cultures were

544 diluted 1:200 into this plate using the same plate seal and grown with shaking at $37^{\circ} \mathrm{C}$

545 in a BioTek Epoch 2 plate reader for 18-24 h. OD 600 was measured at 7.5-min intervals.

546 For the resulting growth curves, the slope over a sliding window (for smoothing) was

547 computed to determine the instantaneous growth rate, and from that, the lag time

548 (defined as the time to half-maximal growth rate) was calculated. Additionally, the 549 natural logarithm of OD 600 was fit to the Gompertz equation [12] to quantify lag time 550 and maximal growth rate. The two methods for calculating lag times and maximum 551 growth rates were highly correlated (Fig. S6) for growth curves of E. coli. The first 552 method was more appropriate for growth curves that did not reach saturation or exhibited a distinct shape (e.g., due to a diauxic shift). Thus, we used the first method to 554 quantify lag time and maximal growth rate, except as noted.

557 Cells were diluted 1:10 or 1:20 (depending on the overnight culture OD) into fresh LB. 558 For time-lapse imaging, $1 \mu \mathrm{L}$ of cell culture was placed onto a large pad (the size of a 
560 depletion in the pad over the course of imaging, because B. subtilis cells lyse in oxygen-

561 limited conditions [8]. The cells were imaged using a Nikon Eclipse Ti-E inverted

562 fluorescence microscope with a 100X (NA 1.40) oil-immersion objective, and integrated

563 using $\mu$ Manager v. 1.3 [61]. Cells were maintained at $37^{\circ} \mathrm{C}$ during imaging with an

564 active-control environmental chamber (HaisonTech).

565

566

567

568

569

570

571

572

573

574

575

576

577

578

579

\section{Strain construction}

We constructed strains to study the role of LTA synthesis in glycerol-induced lag using SPP1 phage transduction [62]. The donor strain was grown for $>6 \mathrm{~h}$ in TY medium (LB supplemented with $0.01 \mathrm{M} \mathrm{MgSO}_{4}$ and $0.1 \mathrm{mM} \mathrm{MnSO}_{4}$ after autoclaving). Ten-fold dilutions of SPP1 phage were added to the culture, and $3 \mathrm{~mL}$ of TY soft $(0.5 \%)$ agar were then mixed with the cell/phage mixture and poured over a TY plate $(1.5 \%$ agar $)$ before overnight incubation. We chose a plate that exhibited nearly complete clearing of cells, thus without many phage-resistant mutants. Five milliliters of TY medium were added to this plate and a 1-mL filter tip was used to scrape up the soft agar. This soft agar/liquid mix was filtered through a $0.4-\mu \mathrm{m}$ filter (Fisher Scientific). The phage were added to a stationary-phase culture (grown for 6-10 h) of the recipient strain $(10 \mu \mathrm{L}$ undiluted phage $+100 \mu \mathrm{L}$ recipient cells, and optionally $900 \mu \mathrm{L}$ TY medium) in TY medium and incubated at $30^{\circ} \mathrm{C}$ for $30 \mathrm{~min}$, then plated onto LB + antibiotic and $0.01 \mathrm{M}$ sodium citrate (sodium citrate was omitted for MLS selection). Plates were incubated 
580 for $24 \mathrm{~h}$ at $30{ }^{\circ} \mathrm{C}$ and transductants were streaked for single colonies to eliminate the

581 phage.

582

The back-crossed transposon mutagenesis strains and deletion strains ( $\Delta \operatorname{sig} X$ and $\Delta d l t A)$ were constructed via double-crossover integration by transformation of genomic DNA using standard transformation procedures [63, 64]. We performed genomic DNA extractions of the original transposon mutagenesis strain, BKK23100 (sigX), and BKK38500 (dltA) using the Promega Wizard kit.

590 The mariner transposon was transduced into the parent strain as described above. The

591 mariner plasmid has a temperature-sensitive origin of replication, a transposase, and a 592 transposon that is marked with kanamycin resistance; the backbone plasmid is marked 593 with MLS resistance [65]. Before transduction of the mariner transposon, the parent 594 strain (CAG74168) was streaked for single colonies on MLS plates at $30^{\circ} \mathrm{C}$. One colony 595 for each library was grown overnight in $3 \mathrm{~mL} \mathrm{LB}+$ kanamycin in a roller drum at room 596 temperature. Transposon-insertion libraries were created by plating 10-fold dilutions of 597 the cultures on $\mathrm{LB}+$ kanamycin plates prewarmed to $37{ }^{\circ} \mathrm{C}$ and incubating them 598 overnight at $37{ }^{\circ} \mathrm{C}$. The dilution that contained very dense colonies was scraped to 
599 create the library, and the higher dilutions were used to estimate the number of

600 transposon mutants per library.

601

602

Transposon library screen

603 The transposon library was screened by using the entire library to inoculate a $5 \mathrm{~mL}$ LB + $6041.25 \%$ glycerol culture. This culture was diluted 1:200 after $18 \mathrm{~h}$ of growth into $200 \mu \mathrm{L}$ of $605 \mathrm{LB}+1.25 \%$ glycerol, grown for $18 \mathrm{~h}$ with shaking at $37^{\circ} \mathrm{C}$, and diluted 1:200 into LB. 606 After two passages of growth in LB + glycerol and one passage in LB, the libraries with 607 a shorter lag time were streaked for single colonies on LB plates. Five single colonies 608 were picked for each positive hit and used to inoculate a 200- $\mu$ L LB culture. After 18 h, 609 the culture was diluted 1:200 into LB $+1.25 \%$ glycerol. The culture was grown for $18 \mathrm{~h}$ 610 and diluted 1:200 into LB. After three passages, the positive hits were grown in $5 \mathrm{~mL}$ of 611 LB for genomic DNA extraction.

612 Mapping transposon insertions using inverse PCR

614 Genomic DNA was extracted using the Promega Wizard Genomic DNA purification 615 kit, digested with Sau3AI at $37^{\circ} \mathrm{C}$ for $90 \mathrm{~min}$, and then heat-inactivated at $65{ }^{\circ} \mathrm{C}$ for 20 616 min. The reaction mixture contained $15.5 \mu \mathrm{L}$ milliQ $\mathrm{H}_{2} \mathrm{O}, 2 \mu \mathrm{L}$ NEBuffer1.1, $2 \mu \mathrm{L}$ 617 digested genomic DNA, $2 \mu \mathrm{L}$ 10X bovine serum albumin, and $0.5 \mu \mathrm{L}$ Sau3AI. The 618 digested DNA was ligated using T4 ligase at room temperature for at least $1 \mathrm{~h}$; the 
619 reaction mixture contained $2 \mu \mathrm{L}$ T4 DNA Ligase Buffer, $15.5 \mu \mathrm{L}$ milliQ $\mathrm{H}_{2} \mathrm{O}, 2 \mu \mathrm{L}$ 620 digested DNA, and $0.5 \mu \mathrm{L}$ T4 DNA ligase. Inverse PCR was carried out with the ligated 621 DNA using Phusion polymerase with the primers IPCR1 (5'622 GCTTGTAAATTCTATCATAATTG-3') and IPCR2 AGGGAATCATTTGAAGGTTGG-3'). Each reaction contained $33 \mu \mathrm{L}$ milliQ $\mathrm{H}_{2} \mathrm{O}, 10 \mu \mathrm{L}$ $6245 X$ HF buffer, $2 \mu \mathrm{L}$ ligated DNA, $2 \mu \mathrm{L}$ IPRC1, $2 \mu \mathrm{L}$ IPCR2, $1 \mu \mathrm{L} 10 \mathrm{mM}$ dNTPs, and 0.2

$625 \mu \mathrm{L}$ Phusion Polymerase. The PCR program was as follows: $98^{\circ} \mathrm{C}$ for $30 \mathrm{~s}, 30$ cycles of $626\left[98{ }^{\circ} \mathrm{C}\right.$ for $10 \mathrm{~s}, 58{ }^{\circ} \mathrm{C}$ for $30 \mathrm{~s}, 72{ }^{\circ} \mathrm{C}$ for $\left.60 \mathrm{~s}\right], 72{ }^{\circ} \mathrm{C}$ for $10 \mathrm{~min}$, and hold at $4{ }^{\circ} \mathrm{C}$. PCR 627 products were gel-purified and sequenced using the IPCR2 primer. The sequences were 628 mapped onto the B. subtilis 168 genome using BLASTN.

631 All data used in this manuscript are growth curves, time-lapse microscopy images, or 632 transposon sequencing. All data are available upon request from the corresponding 633 author. 


\section{Acknowledgments}

635 The authors thank the Huang lab for useful discussions. This work was supported by a

636 Stanford Graduate Fellowship (to E.A.), an NSF Graduate Research Fellowship (to S.C.),

637 an NIH Ruth Kirschstein Award F32-AI133917 (to M.R.), a Bio-X Graduate Fellowship

638 (to A.A.-D.), an HHMI International Student Fellowship (to A.A.-D.), an Agilent

639 Graduate Fellowship (to H.S.), a Stanford Interdisciplinary Graduate Fellowship (to

640 H.S.), a James McDonnell Postdoctoral Fellowship (to H.S.), NSF grant DEB-1951307 (to

641 R.E.L.), USDA Hatch grant MICL02253 (to R.E.L.), NSF CAREER Award MCB-1149328

642 (to K.C.H.), and the Allen Discovery Center at Stanford on Systems Modeling of

643 Infection (to K.C.H.). K.C.H. is a Chan Zuckerberg Biohub Investigator. 
A Day 1

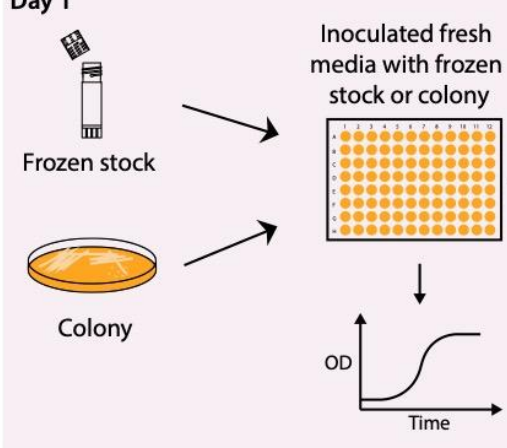

B

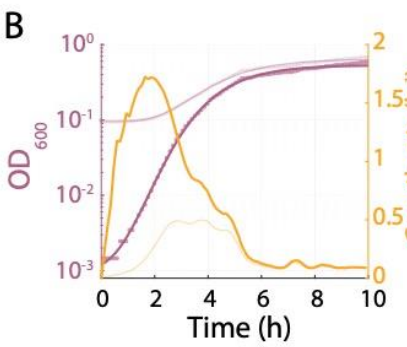

$\mathrm{E}$
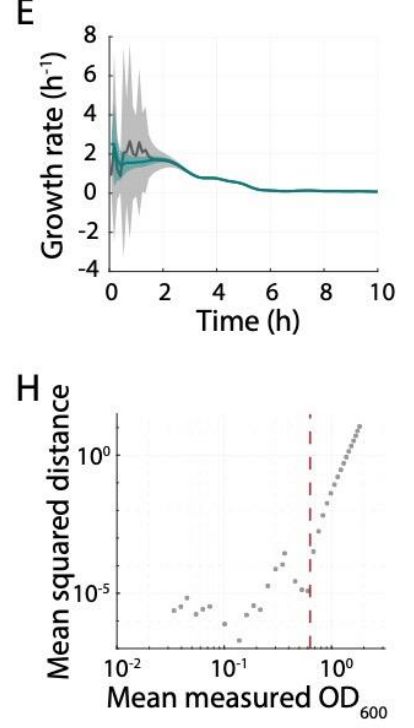

C

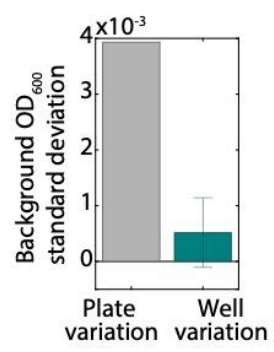

F
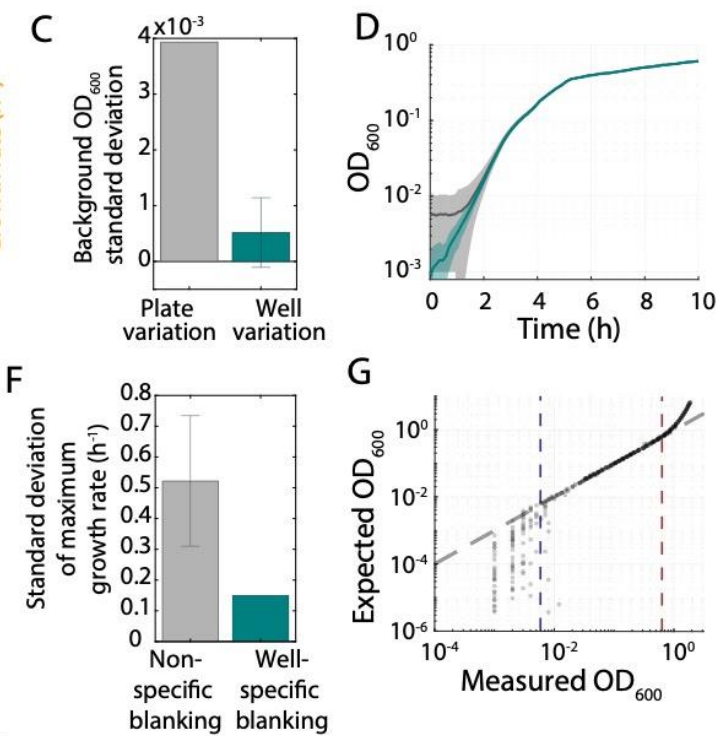

G

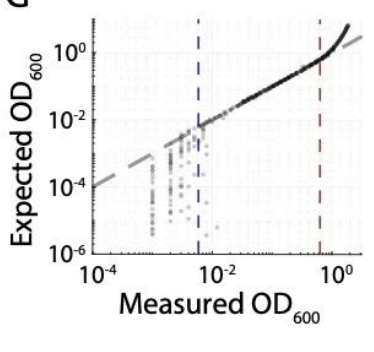

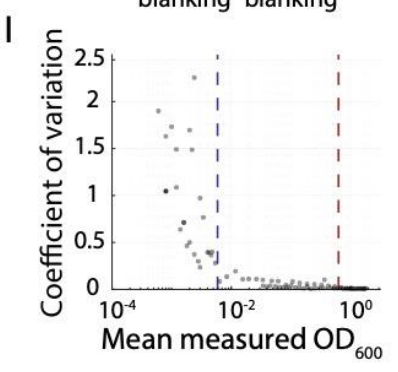

Figure 1: Well-specific blanking and establishment of the spectrophotometer limit of 
B) Raw absorbance values from a typical E. coli MG1655 growth curve (light purple curve) increased starting from just above the background absorbance of the well plus media ( 0.08). Subtracting the background OD resulted in absorbance values (dark purple circles) that indicate substantially different growth kinetics, which are well fit by a Gompertz function (dark purple curve). With background subtraction, the maximum growth rate was 3.7-fold higher and occurs $\sim 60 \mathrm{~min}$ earlier (dark yellow curve) than without subtraction (light yellow curve). the cell-free absorbance of each well (teal) was much lower than when blanking with the background absorbance of a random well (black). Shaded regions represent \pm 1 standard deviation.

E) Instantaneous growth rates computed from growth curves in (B) were much less variable for well-specific blank subtraction (teal) than for blanking with a random well (black). deviation in the estimate of maximum growth rate compared with subtraction of the blank from a randomly selected well in a 96-well plate (gray). 
G) A dilution series from a culture with a known OD can be used to calibrate OD readings in order to establish the range of linearity and the limit of detection of a plate reader. Blue and red dotted lines represent the linear range of detection; even above the linear range the variability is low.

H) The mean squared distance from the line $x=y$ in (D) increased sharply above the red dotted line, justifying its definition as the upper limit of the range of linearity.

I) The coefficient of variation (CV, mean/standard deviation) of OD values in (D) increased sharply below the blue dotted line, justifying its definition as the lower limit of detection. Given the low CV above the linear range (above the red dotted line), it is possible to accurately measure up to an actual OD 3.5 through correction based on the calibration in $(G)$. The red dotted line represents the upper limit of the linear range. 
A

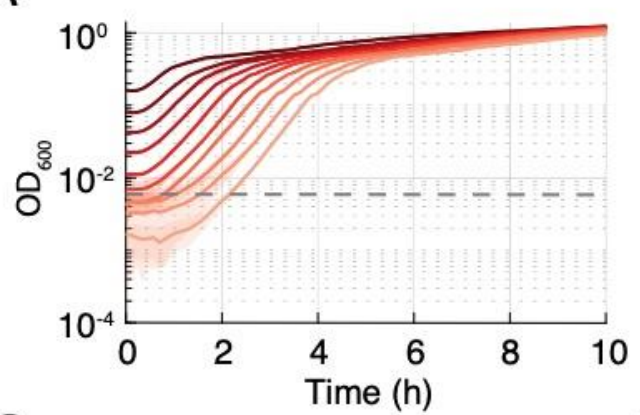

C

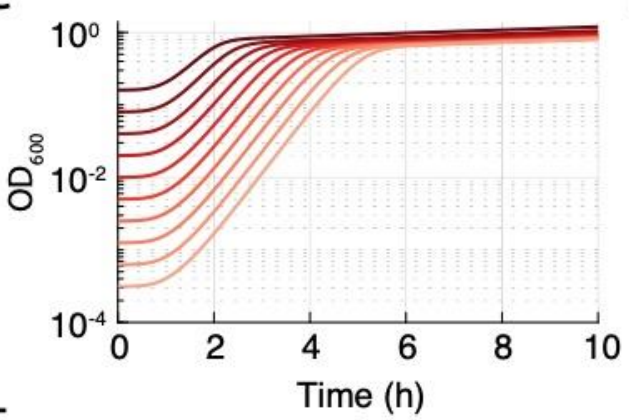

E

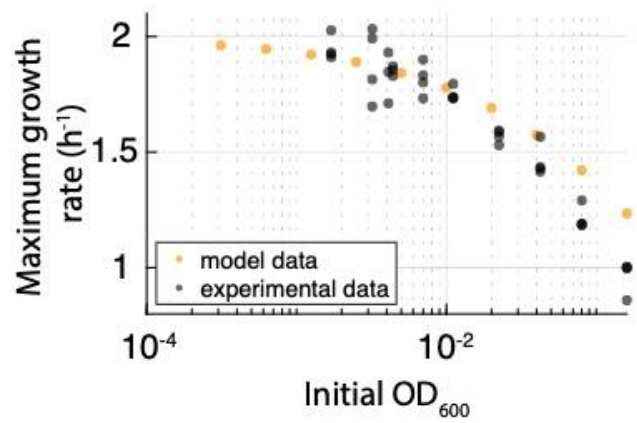

B
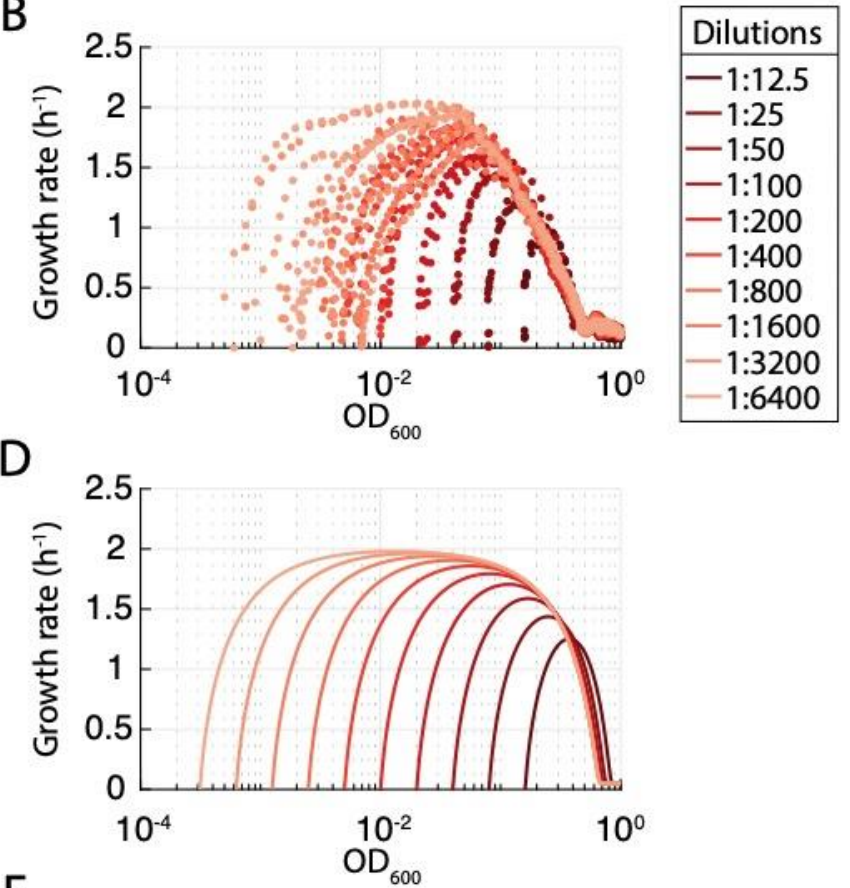

$\mathrm{F}$

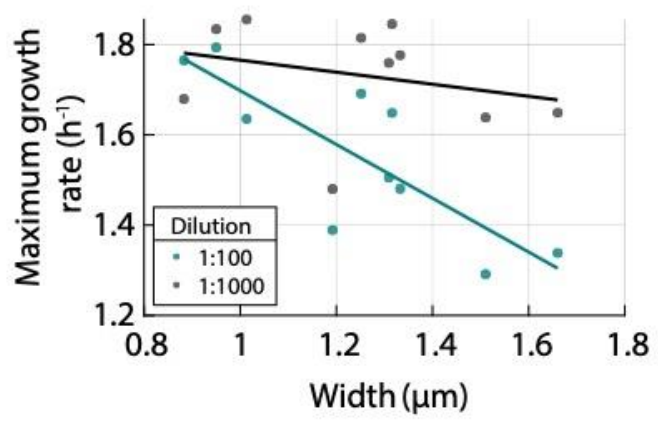

683

Figure 2: Growth rate is intrinsically linked to cell density due to nutrient depletion.

A) Growth curves of a dilution series from a single overnight culture of E. coli MG1655 displayed distinct growth behaviors, with slower initial growth for lower dilutions (higher initial cell density).

B) Instantaneous growth rate as a function of OD for the curves in (B) show that lower dilutions result in lower maximum growth rates. Curves followed a common, approximately linear downward trajectory after reaching maximum 
growth rate, indicating that the entry to stationary phase is less affected by initial OD than lag time or maximum growth rate. Simulated growth curves for a model of growth based on nutrient depletion in Eqs. 4-6 were similar to experimental data in (B). between $\mathrm{OD}$ and instantaneous growth rate. Instantaneous growth rate as a function of $\mathrm{OD}$ for the curves in (C) exhibited similar behaviors to the experimental data in (B).

E) Nutrient depletion was sufficient to recapitulate experimental relationship between initial inoculum size and maximum growth rate. The maximum growth rates of the experimental (B) and simulated growth curves (D) exhibited a quantitatively similar decrease with increasing initial inoculum density. growth curve initialized with a 1:100 dilution of an overnight culture (teal) 
A Day 1

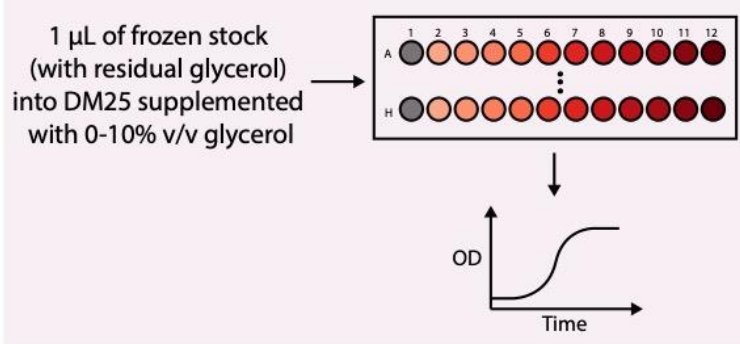

Day 2
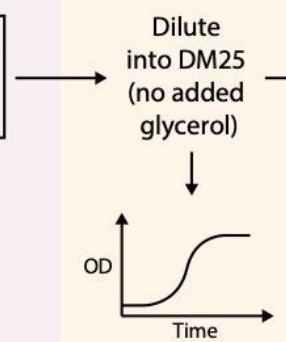

$\rightarrow \begin{gathered}\text { Dilute } \\ \text { into DM25 } \\ \text { (no added }\end{gathered}$

Day 3

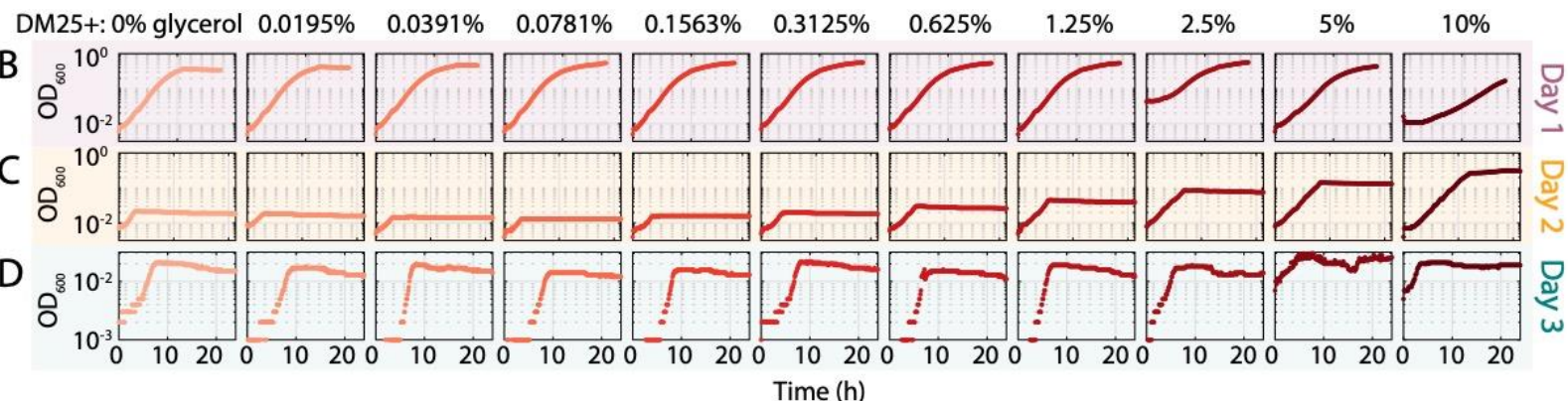

E
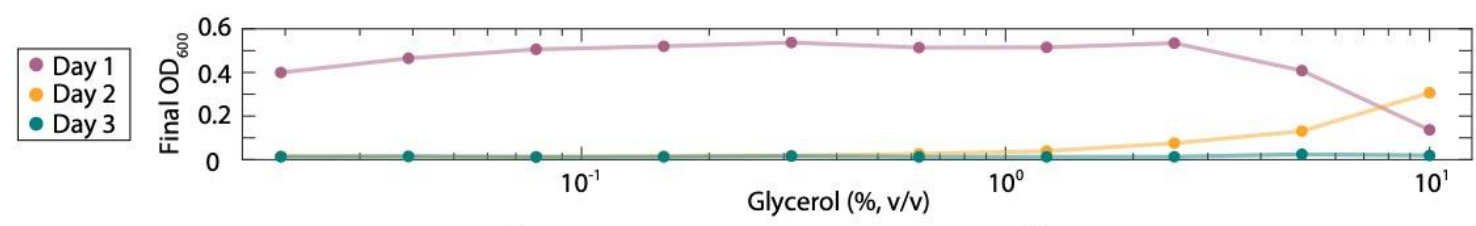

F

G
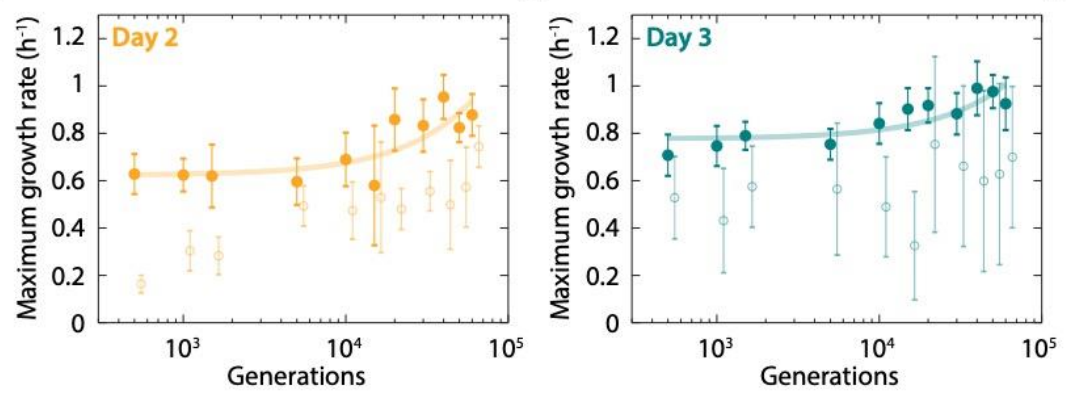

$\mathrm{H}$

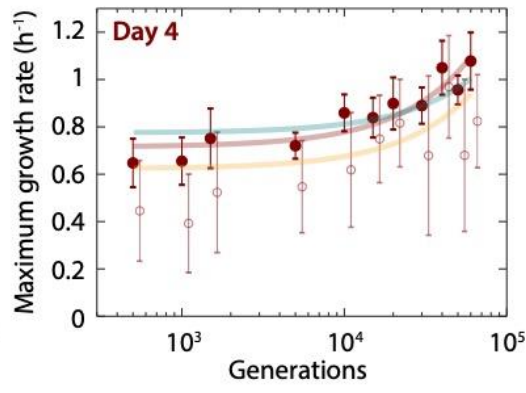

Figure 3: The presence of glycerol in carbon-poor medium increases the carrying

A) Schematic of protocol to measure the effect of glycerol on growth. E. coli cultures 
concentrations of glycerol. Growth was monitored over three passages, in which grown in minimal medium (DM25, $25 \mathrm{mg} / \mathrm{L}$ glucose) with various amounts of glycerol. On day 1, the carrying capacity was much higher than would be remained relatively high following the initially higher glycerol concentrations, presumably due to glycerol carryover.

D) By day 3, growth curves stabilized across all concentrations, and the increase in carrying capacity due to glycerol carryover no longer occurred. Note that the $y$ axis scale is different from those in (B) and (C).

E) Dependence of final OD on glycerol concentration present during growth during the first passage. Final OD was much higher on day 1 compared to day 2 and 3 for all concentrations other than $10 \%$ glycerol. On day 2 , final OD increased with the glycerol concentration from the previous passage. On day 3 , the dependence on glycerol concentration was gone and the final OD was very low, as expected given the carbon-poor medium. 
medium. Maximum growth rates computed using well-specific blanking (filled circles) in DM25 for the Ara-1 evolved line [40] are lower and noisier during the second growth passage (E) than during the third (F) and fourth passages $(\mathrm{G})$. Measurements in $(\mathrm{F})$ and $(\mathrm{G})$ are quantitatively similar and revealed increases in maximum growth over the course of the LTEE. The lines are linear least-squares fits to the data on day 2 (yellow), 3 (teal), and 4 (maroon). Blanking based on a randomly selected well (E-G, open circles) led to generally lower growth-rate estimates and noisier trends; these data are slightly right-shifted to avoid overlap. 


\section{A Day 1}

$1 \mu \mathrm{L}$ of frozen stock (with residual glycerol)
into LB supplemented with $0-10 \% \mathrm{v} / \mathrm{v}$ glycerol

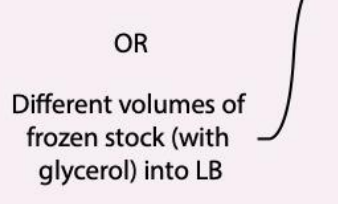

frozen stock (with glycerol) into LB
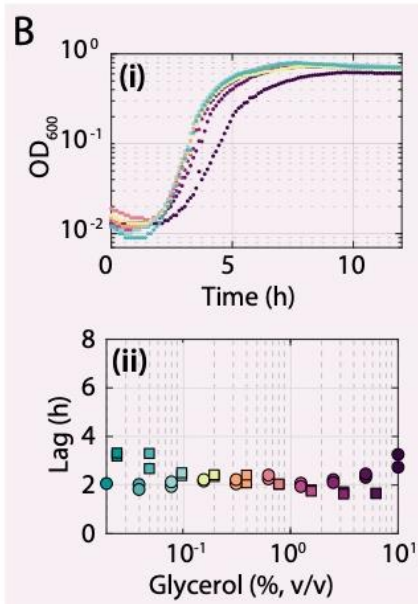
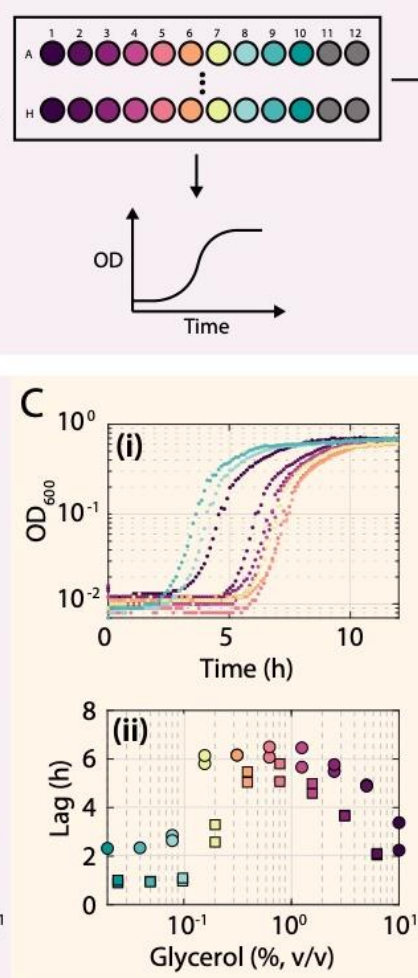

Day 2

Day 3
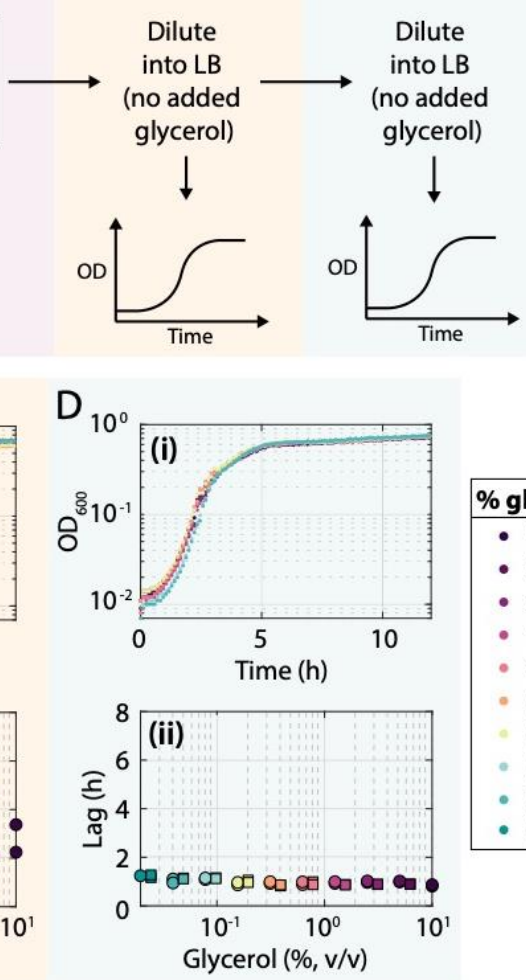

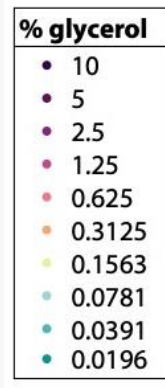

743

Figure 4: Growth with glycerol causes a large increase in lag time for B. subtilis in the

\section{subsequent passage.}

A) Schematic of protocol to measure the effect of glycerol on growth. B. subtilis cultures were either inoculated in LB with various amounts of a frozen stock

(Fig. S3D,E) or $1 \mu \mathrm{L}$ of frozen stock was used to inoculate LB supplemented with various concentrations of glycerol, as shown in (B-D). Growth was monitored over three passages, in which the last two followed 1:200 dilutions into LB (without glycerol). 
glycerol (in addition to the $\sim 0.075 \%$ transferred with the frozen stock). During the second passage $(\mathrm{Ci})$, the cultures had similar maximum growth rates and carrying capacities, but intermediate inoculation amounts led to large increases in lag time. Growth curves were essentially identical during the third passage (Di). On day 1 (Bii), lag times were roughly constant for intermediate inoculation amounts (squares) or glycerol concentrations (circles), but lag times increased dramatically on day 2 (Cii); by day 3 (Dii) there was little difference in lag times across glycerol concentrations. These data indicate that glycerol causes the longvolumes of frozen stock (Fig. S3). 
A

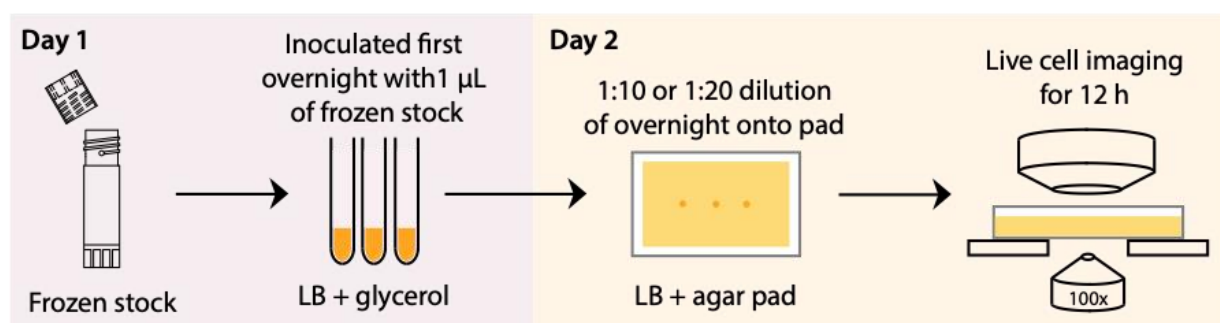

B

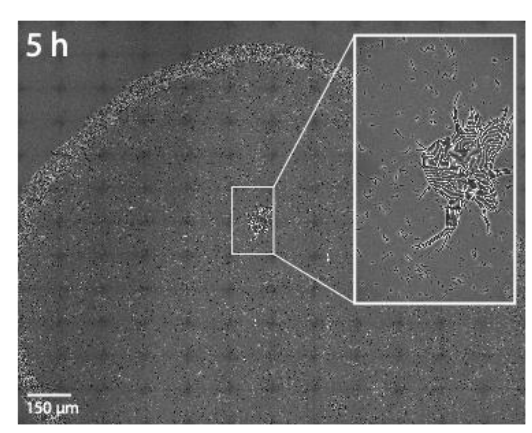

$\mathrm{D}$

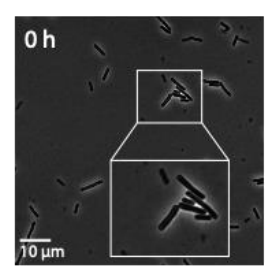

E

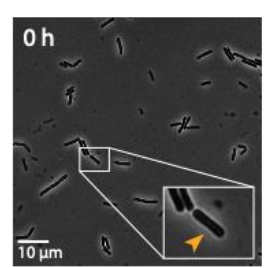

G
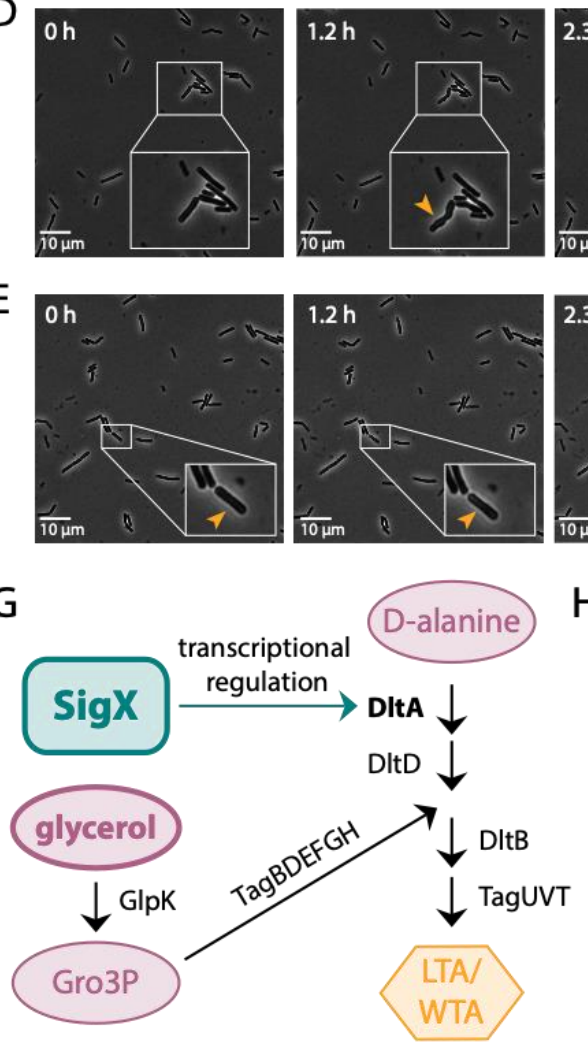
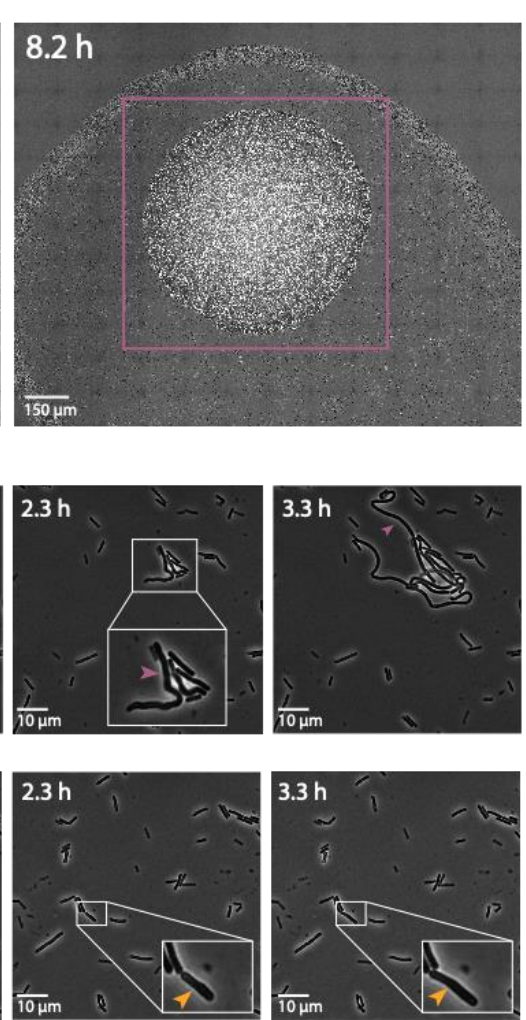

$\mathrm{H}$

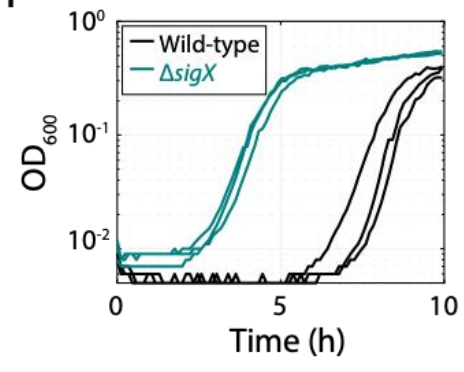

C
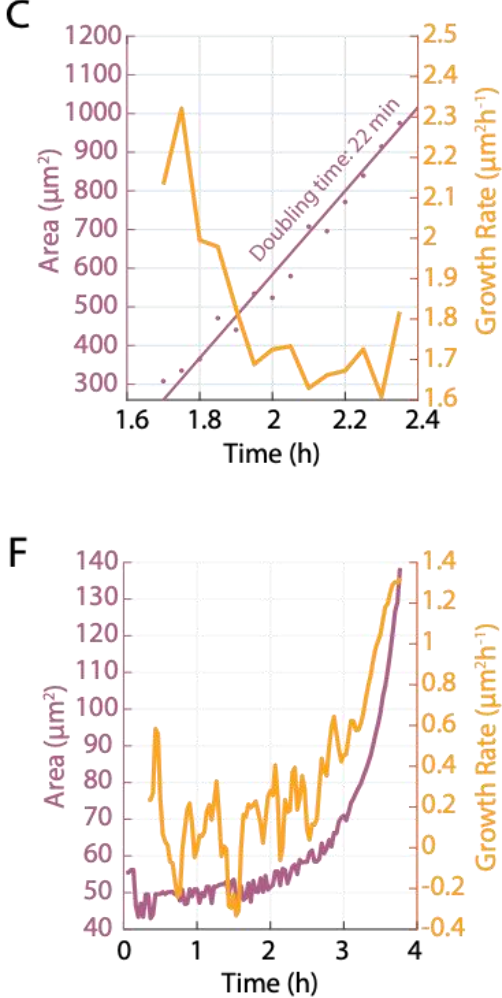

\section{I}

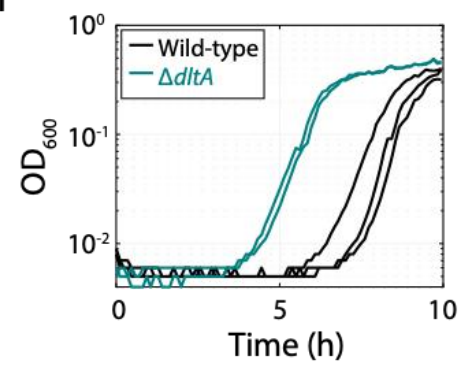


A) Schematic of protocol for imaging single-cell growth during the passage after growth in glycerol. of cells on an LB agarose pad after a passage in LB $+0.3125 \%$ glycerol. Inset, enlarged view of the only cell that exhibited growth across the entire spot. The purple box at right highlights the resulting microcolony at $8.2 \mathrm{~h}$. initially sole growing cell in (B) finds a maximum growth rate corresponding to a doubling time of $22 \mathrm{~min}$.

D) Time-lapse of cell growth on an LB agarose pad after growth in liquid LB + $0.625 \%$ glycerol. The cell (inset, enlarged) started growing immediately, and bulged (yellow arrow) and filamented for $\sim 3 \mathrm{~h}$ (purple arrow). after growth in liquid LB $+0.625 \%$ glycerol; this cell did not start growing until after $\sim 2 \mathrm{~h}$.

F) Quantification of cell area (purple) and instantaneous growth rate (yellow) of the cell in (E), which had a longer lag and slower growth rate than the cell in (C). SigX and DltA. SigX transcriptionally regulates $d l t A$ and 47 other genes, some of which are involved in biosynthesis of teichoic acids. Glycerol is converted to sn- 
Glycerol 3-phosphate (Gro3P); Gro3P, in conjunction with an intermediate product made by DltA, is necessary for the production of teichoic acids. 


\section{Supplementary Figure Legends}

792

793

794

795

796

797

798

799

800

801

802

803

804

805
A

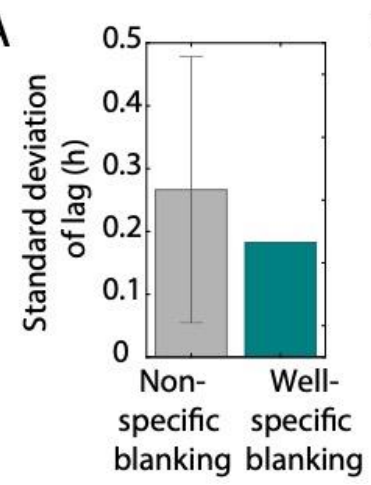

B

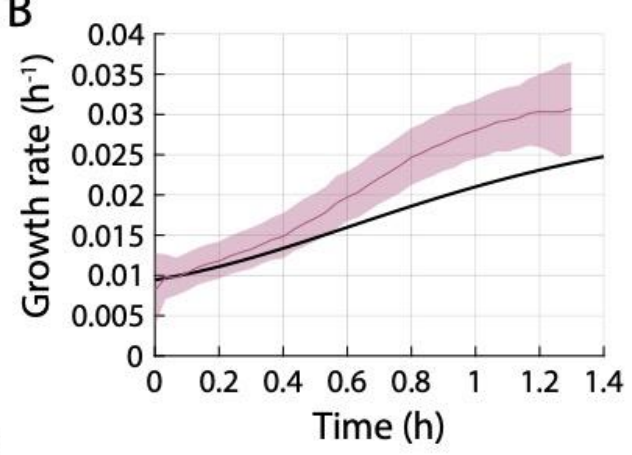

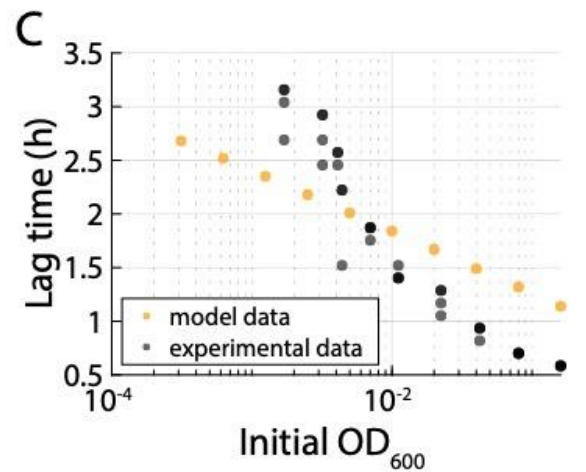

Supplementary Figure 1: Fitting parameters for a model of bacterial population growth that depends on inoculum size, and comparison of this model to experimental data.

A) Well-specific blank subtraction (teal) decreases the standard deviation in the estimate of lag time compared to subtracting a randomly selected blank in a 96well plate (gray).

B) The instantaneous growth rate of E. coli MG1655 cells extracted from single-cell imaging on LB $+1 \%$ agarose pads during emergence from stationary phase increases over the first hour. Shaded region indicates \pm 1 standard deviation. The black line is the Gompertz model with $\lambda=0.8 \mathrm{~h}^{-1}, \delta=0.5 \mathrm{~h}$.

C) Simulated growth curves based on Eqs. 4-6 in the main text capture, at least roughly, the decrease in lag time with increasing initial inoculum density. 
806
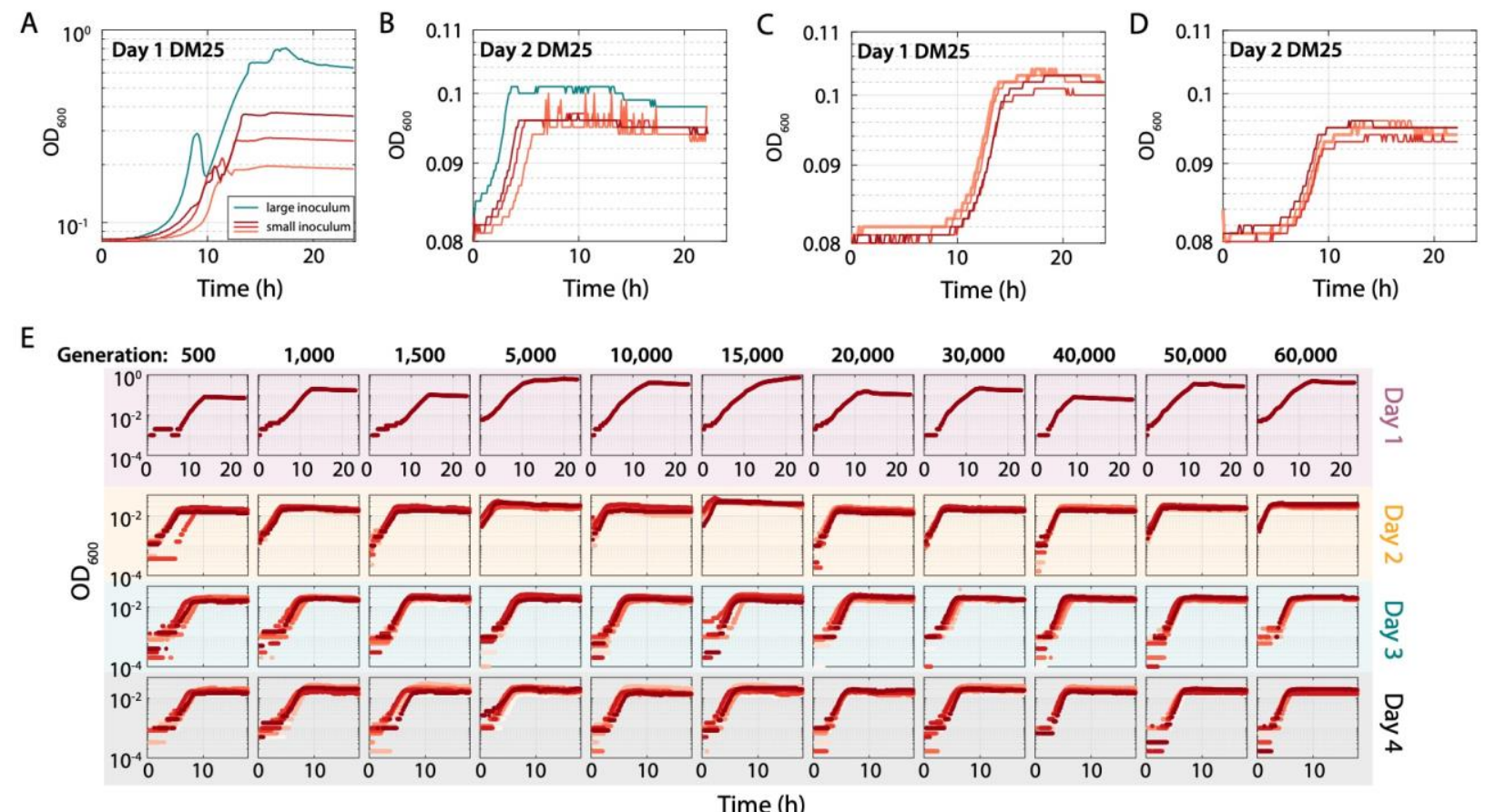

Supplementary Figure 2: Volume from a frozen stock used to inoculate a culture affects carrying capacity in low-resource medium.

A) Various volumes of a frozen stock of E. coli REL606 were used to inoculate cultures grown in DM25. Dark to light red indicate three replicates of a small inoculum size (small but inexact volumes were picked from the frozen stock), while teal indicates an intentionally much larger inoculum volume. The large inoculum (teal) led to a higher final OD on the first day of growth.

B) When the cultures in (A) were diluted 1:200 into fresh DM25 and regrown for 24 $h$, the higher final ODs on day 1 translated into slightly higher ODs (teal) even on day 2. 

frozen stock in $200 \mu \mathrm{L}$ of DM25, spinning down for 5 min at $8000 g$, removing the supernatant, and resuspending the pellet in $200 \mu \mathrm{L}$ of DM25 for growth. The curves represent four technical replicates.

D) When the cultures in (C) were diluted 1:200 into fresh DM25 and regrown for 24 $\mathrm{h}$, the replicates behaved similarly and had a slightly lower final OD than the large inoculum in (B) (teal). 60,000 generations. One microliter of frozen stock (20\% glycerol) of each clone (shown by generation sampled) from the E. coli Ara-1 line [40] was grown in DM25 with various amounts of glycerol across four 24-h passages. On day 1 , the carrying capacity was much higher than was achieved in later days in the lowglucose DM25 medium. The populations were diluted 1:200 on subsequent days into DM25 and growth curves were monitored in a plate reader. The maximum growth rate extracted from the curves is more accurately measured on Days 3 and 4, and it increases over evolutionary time (Fig. 4). All curves are shown after subtracting the blank value of the corresponding well. 


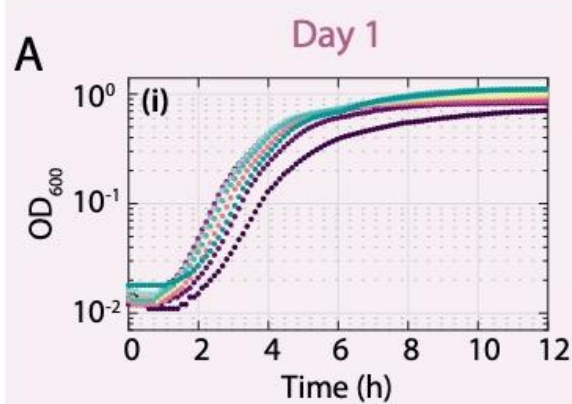

B
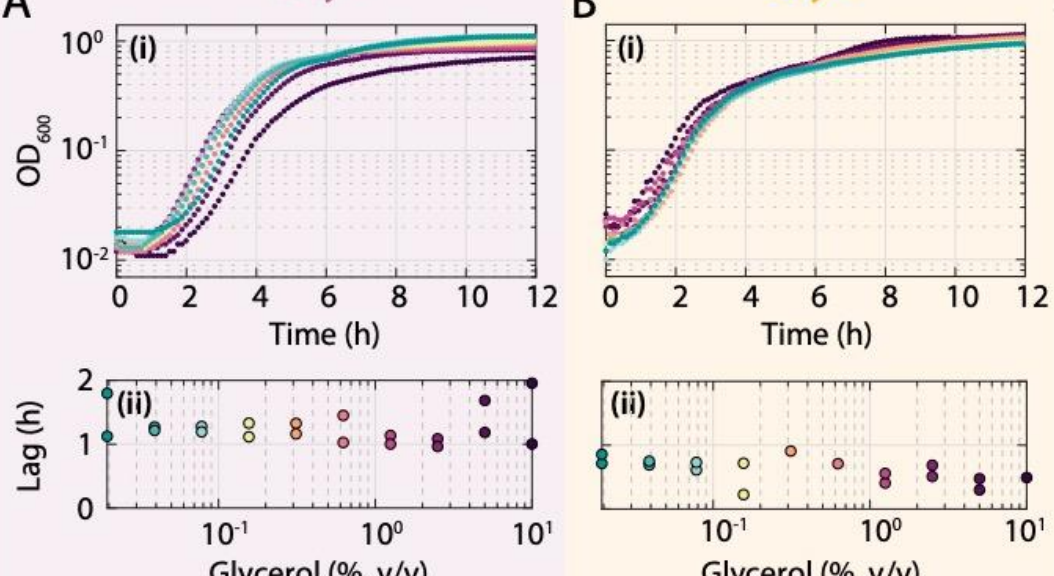

D
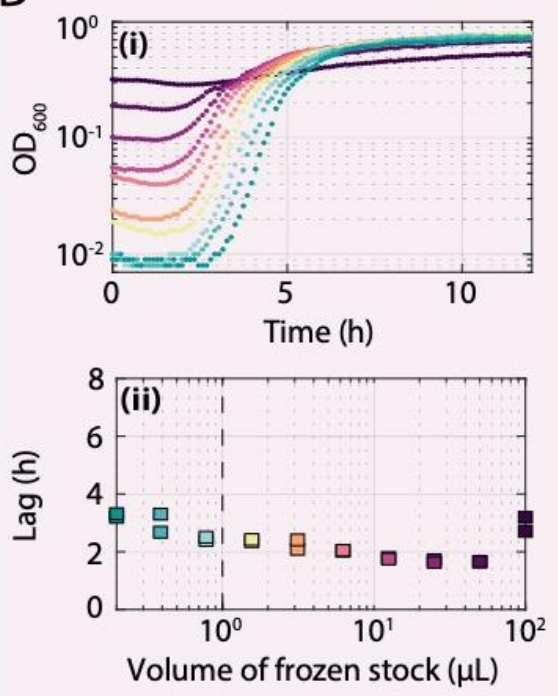

G
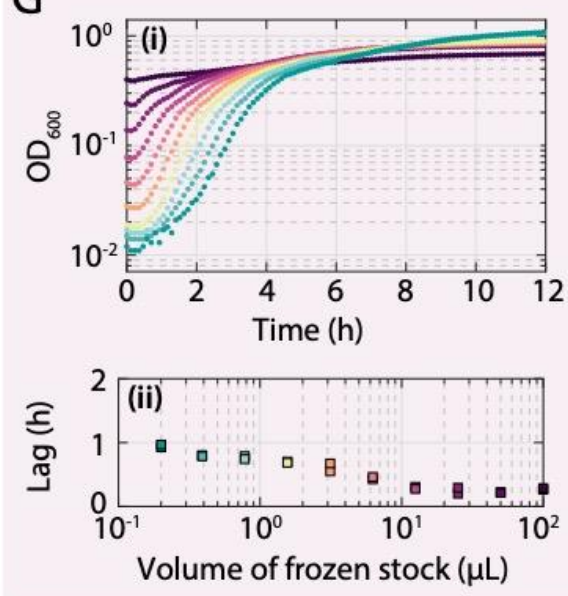

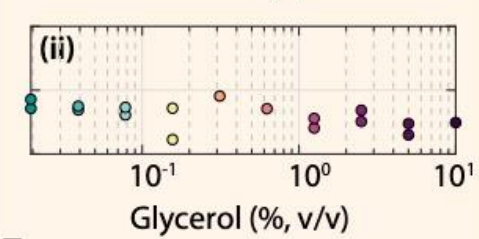

E
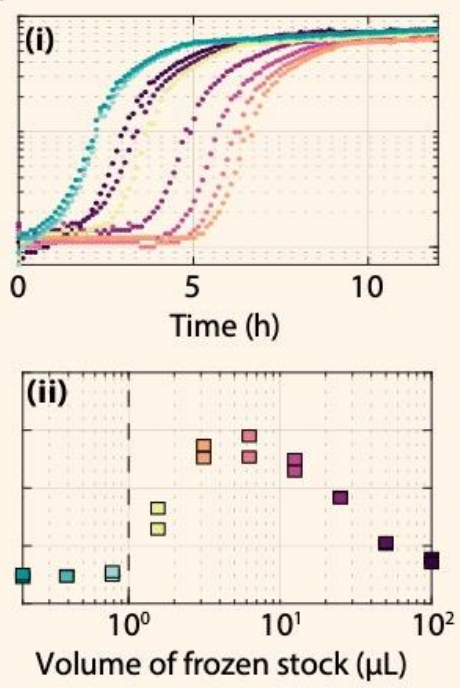

$\mathrm{H}$
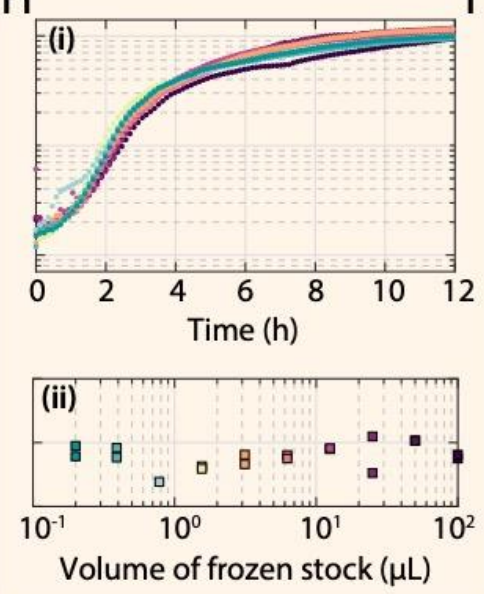
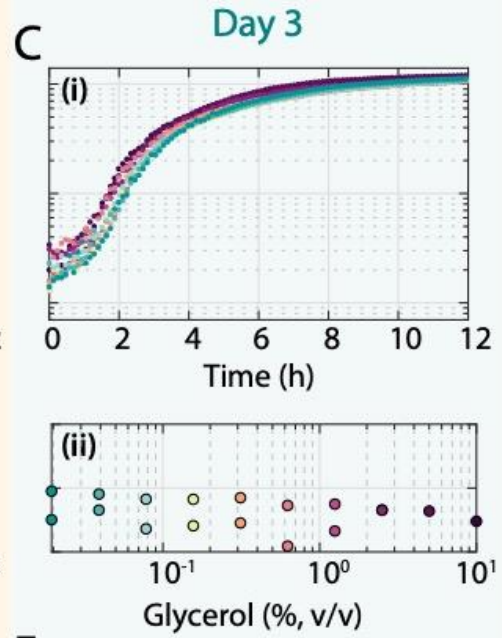

F
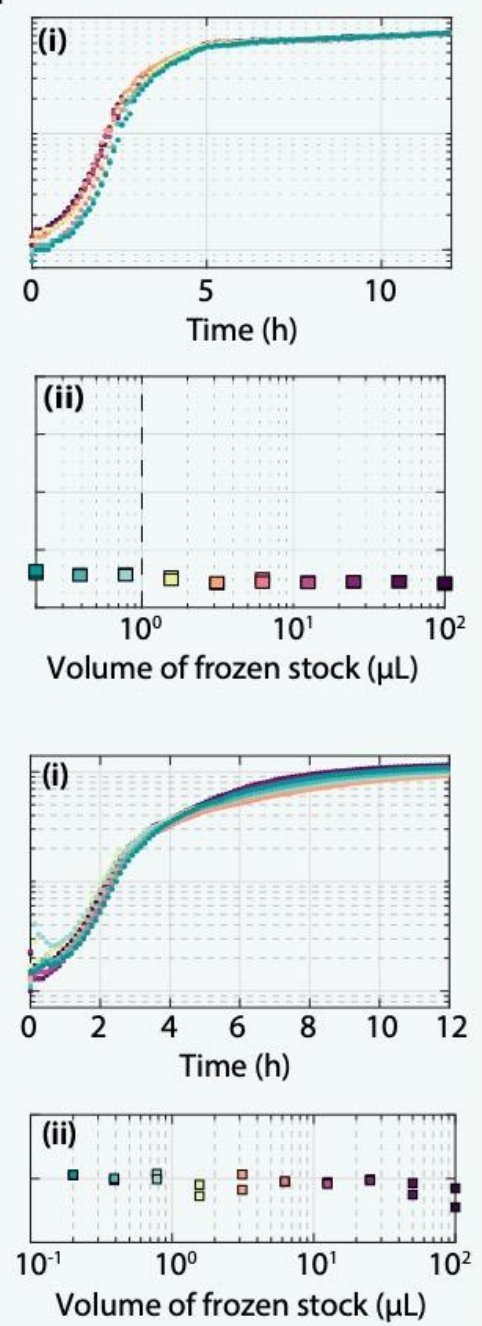
LB. into LB on day $3(\mathrm{Ci})$. There were slight glycerol-dependent effects on day 1 (Ai), but growth curves on days 2 (Bi) and $3(\mathrm{Ci})$ were nearly identical for all cultures. Estimates of lag time from the growth curves are shown in (Aii), (Bii), and (Cii). quantitatively similar across glycerol concentrations. cultures had similar maximum growth rates and carrying capacities, but intermediate inoculation amounts led to much longer lag times Growth curves were essentially identical during the third passage (Fi). Estimates of lag time but they increased dramatically on day 2 (Eii), before becoming uniform and with the increase in lag shown in Fig. $4 \mathrm{C}$. 
854 G-I) Growth curves of E. coli MG1655 in LB supplemented with various volumes of

855 frozen stock on day $1(\mathrm{Gi})$, diluted 1:200 into LB on day 2 (Hi), and diluted again

856 1:200 into LB on day 3 (Ii). Inoculum volume affected initial OD, maximum

857 growth rate, and lag time on day 1 (Gi), but growth curves on day 2 (Hi) and day

8583 (Ii) were nearly identical for all cultures. Estimates of lag time from the growth

859 curves are shown in (Gii), (Hii), and (Iii). Lag times on days 2 (Hii) and 3 (Iii)

$860 \quad$ were quantitatively similar across glycerol concentrations. 
A

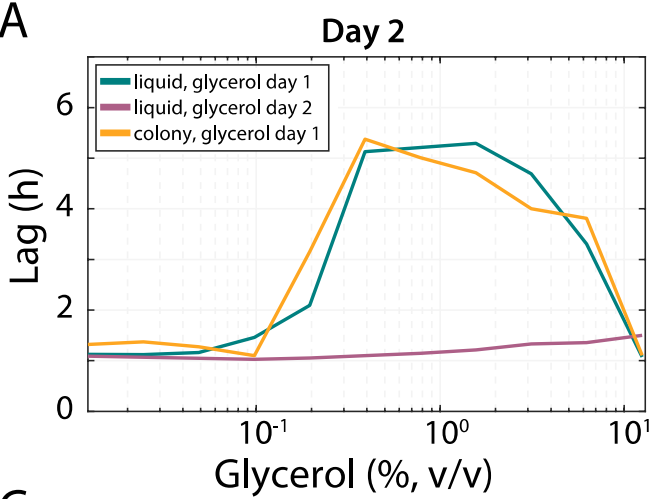

C

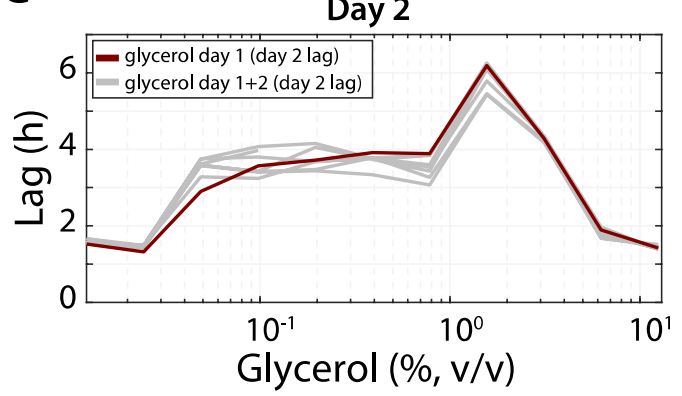

B

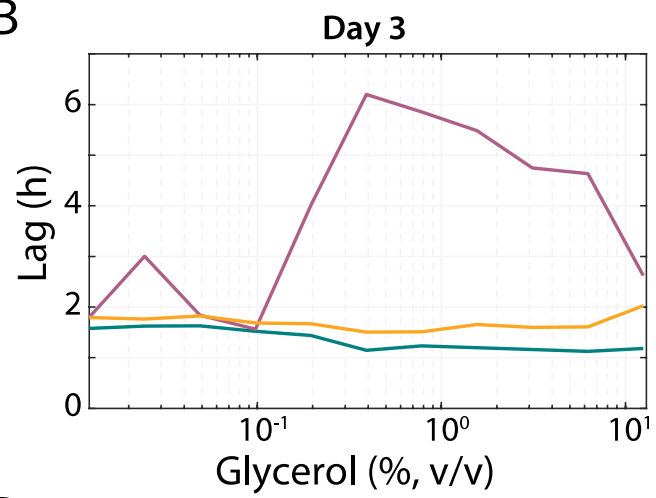

D

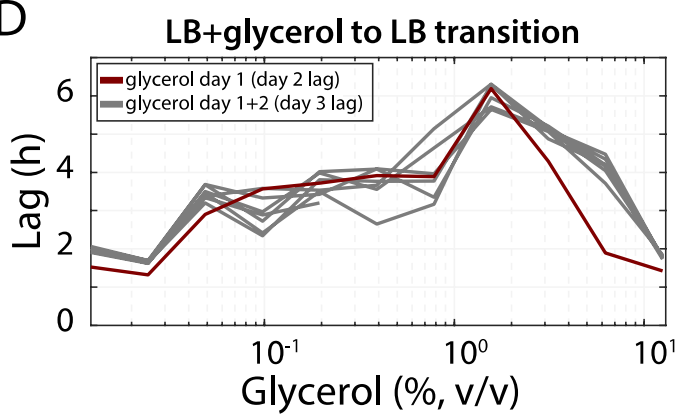

862

863

864

865

866

867

868

869

870

871

872

Supplementary Figure 4: Glycerol causes the long-lag phenotype in B. subtilis.

A) B. subtilis 168 was inoculated from a liquid culture (teal) or a colony (yellow) and grown in LB supplemented with various concentrations of glycerol. In both cases, there were similar lag increases on day 2 after 1:200 dilution and growth in LB. By contrast, there was no long-lag phenotype when cultures (initially inoculated with $1 \mu \mathrm{L}$ of frozen stock on day 1 ) were grown with glycerol on day 2 rather than day 1 (purple).

B) During the next dilution into LB (day 3), the cultures in (A) inoculated with liquid (teal) or colonies (yellow) exhibited short lags, while the glycerol cultures on day 2 (purple) displayed long lags, as expected due to growth in glycerol on the previous day. 
873 C,D) Growth of B. subtilis 168 in LB supplemented with glycerol for two consecutive

874 days caused long lags at intermediate glycerol concentrations on day 2 with glycerol (C, gray) and day 3 without glycerol (D, gray), similar to one day of growth in glycerol (maroon). 
A
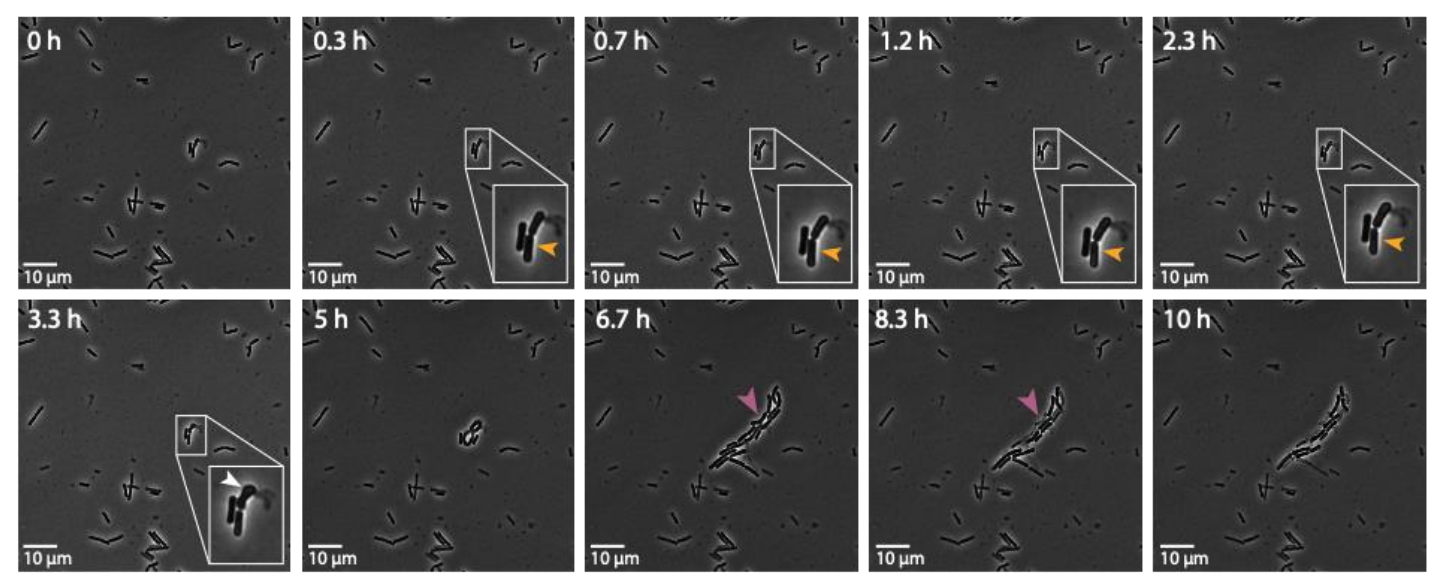

B
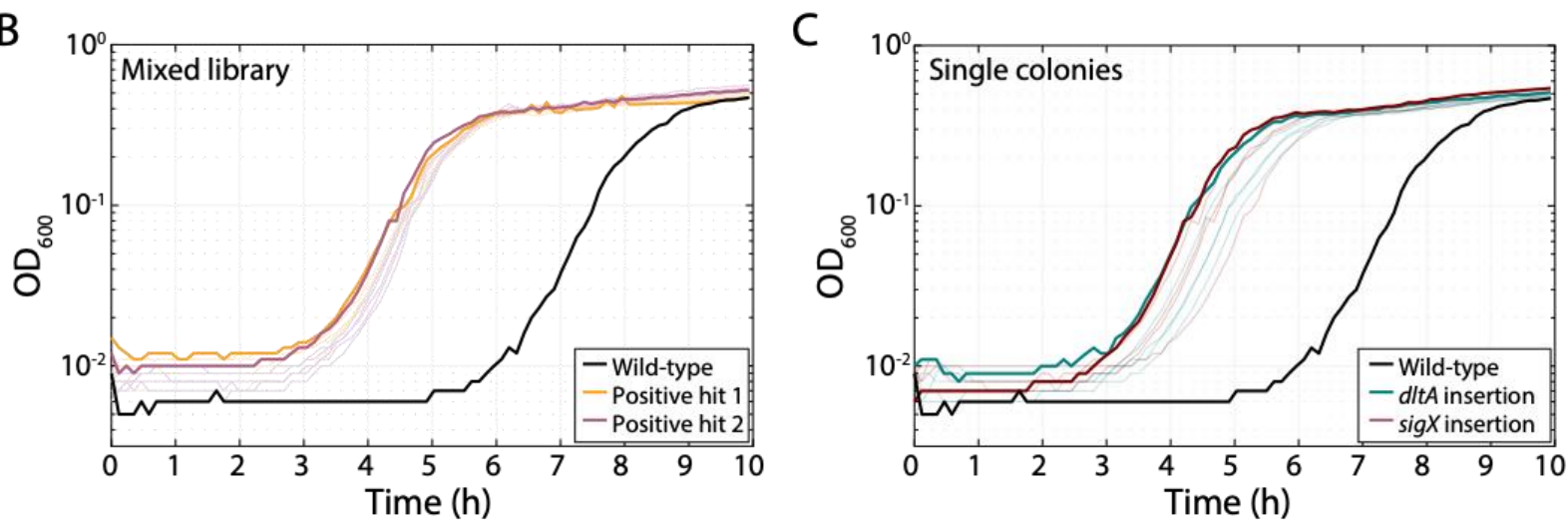

D

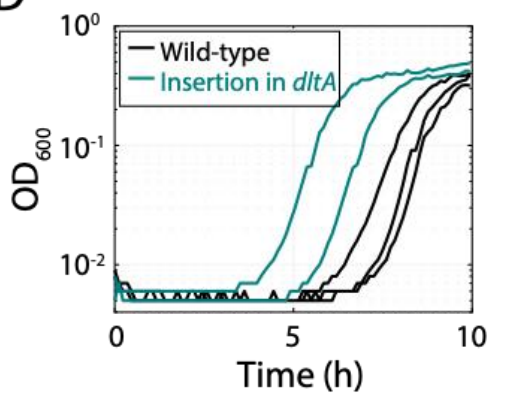

$E$

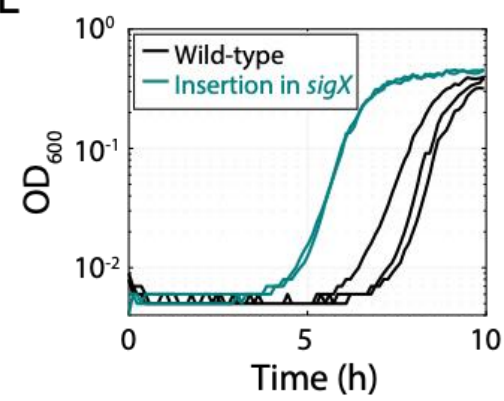

Supplementary Figure 5: Mutations in genes involved in teichoic-acid production

A) Time-lapse images of a cell on an LB agarose pad after growth in liquid LB + $0.625 \%$ glycerol; this cell did not grow for $>3 \mathrm{~h}$, then engaged in short bursts of growth and shrinking (yellow arrows). Small blebs formed on another cell 
during growth (white arrows), and many of its progeny also exhibited periodic shrinking and died by explosive lysis (purple arrows). of libraries with shorter lag times during passaging after growth in LB $+1.25 \%$ glycerol than the parental, wild-type strain (black). Light purple and light yellow curves show 6 biological replicates. passaging after growth in LB $+1.25 \%$ glycerol than the parental, wild-type strain (black). Sequencing revealed that the colonies have insertions in $\operatorname{dlt} A$ (teal) and $\operatorname{sig} X$ (maroon), both of which are linked to lipoteichoic-acid synthesis. Light teal and light maroon curves show 5 biological replicates. 
A

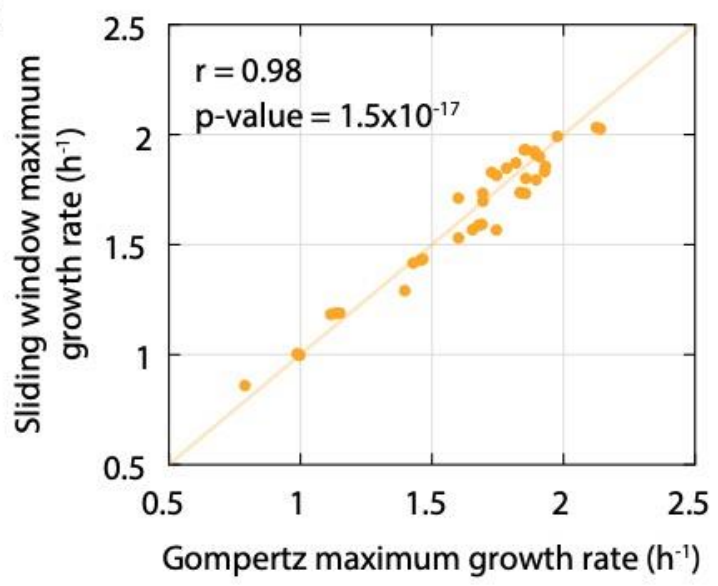

B

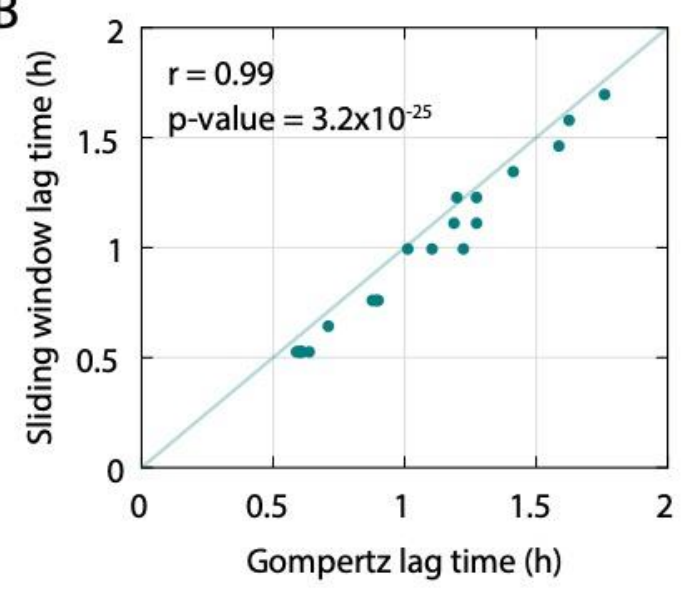

898 Supplementary Figure 6: Fitting a Gompertz function and a linear model to a sliding

A) Maximum growth rates calculated using a Gompertz fit or using a sliding window were highly correlated ( $x=y$ line in yellow). 
906

907

908

909

910

911

912

913

914

915

916

917

918

919

920

921

922

923

924

\section{Movie Legends}

Movie S1: A small fraction of cells exhibit bulging and filamentous growth after overnight passage in LB + 0.625\% glycerol, related to Fig. 5D. Wild-type B. subtilis 168 cells from an 18-h culture in LB $+0.625 \%$ glycerol were spotted onto an LB-agarose pad and imaged every $2 \mathrm{~min}$ at $37^{\circ} \mathrm{C}$. This experiment was done in tandem with Movies S2 and S3.

Movie S2: A small fraction of cells exhibit long lags after overnight passage in LB + $\mathbf{0 . 6 2 5 \%}$ glycerol, related to Fig. 5E. Wild-type B. subtilis 168 cells from an 18-h culture in LB $+0.625 \%$ glycerol were spotted onto an LB-agarose pad and imaged every 2 min at $37^{\circ} \mathrm{C}$. This experiment was done in tandem with Movies S1 and S3.

Movie S3: Some cells exhibit repeated phases of growth and shrinking during regrowth after overnight passage in $\mathrm{LB}+0.625 \%$ glycerol, related to Fig. S5A. Wildtype B. subtilis 168 cells from an 18 -h culture in LB $+0.625 \%$ glycerol were spotted onto an LB-agarose pad and imaged every $2 \mathrm{~min}$ at $37^{\circ} \mathrm{C}$. For one cell, no growth was observed for the first $3 \mathrm{~h}$; afterward it engaged in short phases of growth and shrinking, with small blebs forming during growth. Some of its progeny also exhibited periodic 
bioRxiv preprint doi: https://doi.org/10.1101/2020.06.16.156182; this version posted June 18, 2020. The copyright holder for this preprint (which was not certified by peer review) is the author/funder. All rights reserved. No reuse allowed without permission.

925 shrinking and death through explosive lysis. This experiment was done in tandem with

926 Movies S1 and S2. 


\section{Supplementary Tables}

\begin{tabular}{|c|c|c|c|}
\hline $\begin{array}{c}\text { Strain } \\
\text { designation }\end{array}$ & $\begin{array}{l}\text { Alternative } \\
\text { name }\end{array}$ & Genotype or source name & $\begin{array}{c}\text { Reference, } \\
\text { source, or } \\
\text { both }\end{array}$ \\
\hline EA32 & MG1655 & E. coli K-12: $\mathrm{F}^{-} \lambda^{-} i l v G^{-} r f b-50 r p h-1$ & $\begin{array}{l}\text { KC Huang's } \\
\text { lab }\end{array}$ \\
\hline EA33 & REL606 & $\begin{array}{l}\text { E. coli B: } \mathrm{F}^{-}, \text {tsx-467(Am), araA-, lon-, } \\
\text { rpsL227(strR), hsdR-, }\left[\mathrm{mal}^{+}\right]\left(\mathrm{LamS}^{-}\right)\end{array}$ & $\begin{array}{l}\text { [37], Richard } \\
\text { Lenski's lab }\end{array}$ \\
\hline EA34 & Ara-1 $0.5 \mathrm{k}$ & LTEE strain REL762B & $\begin{array}{l}\text { [41], Richard } \\
\text { Lenski's lab }\end{array}$ \\
\hline EA35 & Ara-1 1k & LTEE strain REL964B & $\begin{array}{l}\text { [41], Richard } \\
\text { Lenski's lab }\end{array}$ \\
\hline EA36 & Ara-1 1.5k & LTEE strain REL1068B & $\begin{array}{l}\text { [41], Richard } \\
\text { Lenski's lab }\end{array}$ \\
\hline EA37 & Ara-1 2k & LTEE strain REL1164A & $\begin{array}{l}\text { [41], Richard } \\
\text { Lenski's lab }\end{array}$ \\
\hline EA38 & Ara-1 5k & LTEE strain REL2179A & $\begin{array}{l}\text { [41], Richard } \\
\text { Lenski's lab }\end{array}$ \\
\hline EA39 & Ara-1 10k & LTEE strain REL4536A & $\begin{array}{l}\text { [41], Richard } \\
\text { Lenski's lab }\end{array}$ \\
\hline EA40 & Ara-1 15k & LTEE strain REL7177A & $\begin{array}{l}\text { [41], Richard } \\
\text { Lenski's lab }\end{array}$ \\
\hline EA41 & Ara-1 20k & LTEE strain REL8593A & $\begin{array}{l}\text { [41], Richard } \\
\text { Lenski's lab }\end{array}$ \\
\hline EA42 & Ara-1 30k & LTEE strain REL10391 & $\begin{array}{l}\text { [41], Richard } \\
\text { Lenski's lab }\end{array}$ \\
\hline EA43 & Ara-1 40k & LTEE strain REL10938 & $\begin{array}{l}\text { [41], Richard } \\
\text { Lenski's lab }\end{array}$ \\
\hline EA44 & Ara-1 50k & LTEE strain REL11330 & $\begin{array}{l}\text { [41], Richard } \\
\text { Lenski's lab }\end{array}$ \\
\hline
\end{tabular}




\begin{tabular}{|c|c|c|c|}
\hline EA45 & Ara-1 60k & LTEE strain REL11691 & $\begin{array}{l}\text { Richard } \\
\text { Lenski's lab }\end{array}$ \\
\hline EA46 & 168 & B. subtilis: $\operatorname{trpC2}$ & $\begin{array}{l}\text { Carol Gross's } \\
\text { lab }\end{array}$ \\
\hline \multicolumn{4}{|c|}{ Back-crossed transposon mutagenesis strains } \\
\hline EA47 & $d l t A$ & 168, $\triangle S P B, \triangle P B S X, \triangle p B S 32$, dltA::pMarA-kan & This work \\
\hline EA48 & $\operatorname{sig} X$ & $168, \triangle S P B, \triangle P B S X, \triangle p B S 32, \operatorname{sigX}:: p M a r A-k a n$ & This work \\
\hline \multicolumn{4}{|c|}{ Deletions of transposon mutagenesis strains } \\
\hline BKK38500 & $\Delta d l t A$ & 168 , dltA::kan & [24] \\
\hline BKK23100 & $\Delta \operatorname{sig} X$ & 168, sigX::kan & {$[24]$} \\
\hline EA49 & $\triangle d l t A$ & 168, dltA::kan & This work \\
\hline EA50 & $\Delta \operatorname{sig} X$ & $168, \operatorname{sig} X:: k a n$ & This work \\
\hline
\end{tabular}


929

930

931

932

933

934

935

936

937

938

939

940

941

942

943

944

945

946

947

\section{References}

1. Weinstock, M.T., et al., Vibrio natriegens as a fast-growing host for molecular biology.

Nat Methods, 2016. 13(10): p. 849-51.

2. Jørgensen, B.B. and S. Hondt, A Starving Majority Deep Beneath the Seafloor.

Science, 2006. 314(5801): p. 932.

3. Roszak, D.B. and R.R. Colwell, Survival strategies of bacteria in the natural

environment. Microbiological reviews, 1987. 51(3): p. 365-379.

4. Monds, R.D., et al., Systematic perturbation of cytoskeletal function reveals a linear scaling relationship between cell geometry and fitness. Cell Rep, 2014. 9(4): p. 1528-37.

5. Peters, J.M., et al., A Comprehensive, CRISPR-based Functional Analysis of Essential Genes in Bacteria. Cell, 2016. 165(6): p. 1493-1506.

6. Vasi, F., M. Travisano, and R.E. Lenski, Long-term experimental evolution in Escherichia coli. II. Changes in life-history traits during adaptation to a seasonal environment. The american naturalist, 1994. 144(3): p. 432-456.

7. Monod, J., The Growth of Bacterial Cultures. Annual Review of Microbiology, 1949. 3: p. 371-394.

8. Arjes, H.A., et al., Biosurfactant-Mediated Membrane Depolarization Maintains Viability during Oxygen Depletion in Bacillus subtilis. Current Biology, 2020. 30(6): p. 1011-1022.e6. 
948

949

950

951

952

953

954

955

956

957

958

959

960

961

962

963

964

965

966

967

9. Arjes, Heidi A., et al., Failsafe Mechanisms Couple Division and DNA Replication in Bacteria. Current Biology, 2014. 24(18): p. 2149-2155.

10. Koch, A.L., Some calculations on the turbidity of mitochondria and bacteria.

Biochimica et Biophysica Acta, 1961. 51(3): p. 429-441.

11. Koch, A.L., Turbidity measurements of bacterial cultures in some available commercial instruments. Analytical Biochemistry, 1970. 38(1): p. 252-259.

12. Zwietering, M.H., et al., Modeling of the Bacterial Growth Curve. Applied and Environmental Microbiology, 1990. 56(6): p. 1875-1881.

13. Tonner, P.D., et al., Detecting differential growth of microbial populations with Gaussian process regression. Genome research, 2017. 27(2): p. 320-333.

14. Baranyi, J. and T.A. Roberts, A dynamic approach to predicting bacterial growth in food. International Journal of Food Microbiology, 1994. 23(3): p. 277-294.

15. Gibson, A.M., N. Bratchell, and T.A. Roberts, Predicting microbial growth: growth responses of salmonellae in a laboratory medium as affected by $\mathrm{pH}$, sodium chloride and storage temperature. International Journal of Food Microbiology, 1988. 6(2): p. 155178.

16. Hills, B.P. and K.M. Wright, A New Model for Bacterial Growth in Heterogeneous Systems. Journal of Theoretical Biology, 1994. 168(1): p. 31-41.

17. Jones, J.E., et al., Mathematical modelling of the growth, survival and death of Yersinia enterocolitica. International Journal of Food Microbiology, 1994. 23(3): p. 433-447. 
968

969

970

971

972

973

974

975

976

977

978

979

980

981

982

983

984

985

986

987

18. Whiting, R.C. and J.E. Call, Time of growth model for proteolytic Clostridium

botulinum. Food Microbiology, 1993. 10(4): p. 295-301.

19. Whiting, R.C. and M. Cygnarowicz-Provost, A quantitative model for bacterial growth and decline. Food Microbiology, 1992. 9(4): p. 269-277.

20. Schepers, A.W., J. Thibault, and C. Lacroix, Comparison of simple neural networks and nonlinear regression models for descriptive modeling of Lactobacillus helveticus growth in pH-controlled batch cultures. Enzyme and Microbial Technology, 2000. 26(5): p. 431-445.

21. McKellar, R.C., A heterogeneous population model for the analysis of bacterial growth kinetics. International Journal of Food Microbiology, 1997. 36(2): p. 179-186.

22. Blount, Z.D., et al., Genomic analysis of a key innovation in an experimental Escherichia coli population. Nature, 2012. 489(7417): p. 513-8.

23. Baba, T., et al., Construction of Escherichia coli K-12 in-frame, single-gene knockout mutants: the Keio collection. Mol Syst Biol, 2006. 2: p. 20060008.

24. Koo, B.M., et al., Construction and Analysis of Two Genome-Scale Deletion Libraries for Bacillus subtilis. Cell Syst, 2017. 4(3): p. 291-305 e7.

25. Campos, M., et al., Genomewide phenotypic analysis of growth, cell morphogenesis, and cell cycle events in Escherichia coli. Mol Syst Biol, 2018. 14(6): p. e7573.

26. Auer, G.K., et al., Mechanical Genomics Identifies Diverse Modulators of Bacterial Cell Stiffness. Cell Syst, 2016. 2(6): p. 402-11. 
988

989

990

991

992

993

994

995

996

997

998

999

1000

1001

1002

1003

1004

1005

1006
27. Trivedi, R.R., et al., Mechanical Genomic Studies Reveal the Role of Alanine

Metabolism in Pseudomonas aeruginosa Cell Stiffness. mBio, 2018. 9.

28. Liu, H., et al., Magic Pools: Parallel Assessment of Transposon Delivery Vectors in Bacteria. mSystems, 2018. 3(1).

29. Shiver, A.L., et al., Rapid ordering of barcoded transposon insertion libraries of anaerobic bacteria. bioRxiv, 2019.

30. Liu, H., et al., Large-scale chemical-genetics of the human gut bacterium Bacteroides thetaiotaomicron. bioRxiv, 2019.

31. Colavin, A., H. Shi, and K.C. Huang, RodZ modulates geometric localization of the bacterial actin MreB to regulate cell shape. Nat Commun, 2018. 9(1): p. 1280.

32. Schmidt, A., et al., The quantitative and condition-dependent Escherichia coli proteome. Nat Biotechnol, 2016. 34(1): p. 104-10.

33. Gompertz, B., On the Nature of the Function Expressive of the Law of Human Mortality, and on a New Mode of Determining the Value of Life Contingencies.

Philosophical Transactions of the Royal Society of London, 1825. 115: p. 513-583.

34. Shi, H., et al., Deep Phenotypic Mapping of Bacterial Cytoskeletal Mutants Reveals Physiological Robustness to Cell Size. Curr Biol, 2017. 27(22): p. 3419-3429 e4.

35. Feltham, R.K.A., et al., A Simple Method for Storage of Bacteria at $-76^{\circ} \mathrm{C}$. Journal of Applied Bacteriology, 1978. 44(2): p. 313-316. 
1007

1008

1009

1010

1011

1012

1013

1014

1015

1016

1017

1018

1019

1020

1021

1022

1023

1024

1025

1026

36. Perry, S.E., Freeze-Drying and Cryopreservation of Bacteria in Methods in Molecular Biology: Cryopreservaflon and Freeze-Drying Protocols J.G. Day and M.R. Mclellan, Editors. 1995, Humana Press Inc.: Totowa, NJ p. 21-30.

37. Lenski, R.E., et al., Long-Term Experimental Evolution in Escherichia coli. I. Adaptation and Divergence During 2,000 Generations. The American Naturalist, 1991. 138(6): p. 1315-1341.

38. Studier, F.W., et al., Understanding the differences between genome sequences of Escherichia coli B strains REL606 and BL21(DE3) and comparison of the E. coli B and K-12 genomes. J Mol Biol, 2009. 394(4): p. 653-80.

39. Wiser, M.J., N. Ribeck, and R.E. Lenski, Long-term dynamics of adaptation in asexual populations. Science, 2013. 342(6164): p. 1364-7.

40. Good, B.H., et al., The dynamics of molecular evolution over 60,000 generations. Nature, 2017. 551(7678): p. 45-50.

41. Tenaillon, O., et al., Tempo and mode of genome evolution in a 50,000-generation experiment. Nature, 2016. 536(7615): p. 165-70.

42. Novak, M., et al., Experimental tests for an evolutionary trade-off between growth rate and yield in E. coli. Am Nat, 2006. 168(2): p. 242-51.

43. Singh, K.D., et al., Carbon catabolite repression in Bacillus subtilis: quantitative analysis of repression exerted by different carbon sources. J Bacteriol, 2008. 190(21): p. 7275-84. 
1027

1028

1029

1030

1031

1032

1033

1034

1035

1036

1037

1038

1039

1040

1041

1042

1043

1044

1045

1046

44. Petit, M.A., et al., Tn10-derived transposons active in Bacillus subtilis. Journal of bacteriology, 1990. 172(12): p. 6736-6740.

45. Cao, M. and J.D. Helmann, The Bacillus subtilis extracytoplasmic-function sigmaX factor regulates modification of the cell envelope and resistance to cationic antimicrobial peptides. J Bacteriol, 2004. 186(4): p. 1136-46.

46. Heaton, M.P. and F.C. Neuhaus, Biosynthesis of D-alanyl-lipoteichoic acid: cloning, nucleotide sequence, and expression of the Lactobacillus casei gene for the D-alanineactivating enzyme. Journal of Bacteriology, 1992. 174(14): p. 4707.

47. Neuhaus, F.C. and J. Baddiley, A continuum of anionic charge: structures and functions of D-alanyl-teichoic acids in gram-positive bacteria. Microbiol Mol Biol Rev, 2003. 67(4): p. 686-723.

48. Wormann, M.E., et al., Enzymatic activities and functional interdependencies of Bacillus subtilis lipoteichoic acid synthesis enzymes. Mol Microbiol, 2011. 79(3): p. 566-83.

49. Parker, R.F., Action of penicillin on Staphylococcus; effect of size of inoculum on the test for sensitivity. Proceedings of the Society for Experimental Biology and Medicine. Society for Experimental Biology and Medicine (New York, N.Y.), 1946. 63(2): p. 443-446.

50. Stevenson, K., et al., General calibration of microbial growth in microplate readers. Sci Rep, 2016. 6: p. 38828. 
1047 51. Aranda-Diaz, A., et al., Bacterial interspecies interactions modulate $p H$-mediated antibiotic tolerance. Elife, 2020.9.

52. Blount, Z.D., C.Z. Borland, and R.E. Lenski, Historical contingency and the evolution of a key innovation in an experimental population of Escherichia coli. Proc Natl Acad

53. Grosskopf, T., et al., Metabolic modelling in a dynamic evolutionary framework predicts adaptive diversification of bacteria in a long-term evolution experiment. BMC

54. Maddamsetti, R., R.E. Lenski, and J.E. Barrick, Adaptation, Clonal Interference, and

55. Rozen, D.E. and R.E. Lenski, Long-Term Experimental Evolution in Escherichia coli. generation experiment with bacterial populations. Proceedings of the National

57. Schaechter, M., O. MaalØe, and N.O. Kjeldgaard, Dependency on Medium and 
1066

1067

1068

1069

1070

1071

1072

1073

1074

1075

1076

1077

1078

1079

1080

1081

1082

1083

1084

1085

58. Mongold, J.A. and R.E. Lenski, Experimental rejection of a nonadaptive explanation for increased cell size in Escherichia coli. J Bacteriol, 1996. 178(17): p. 5333-4.

59. Schirner, K., et al., Distinct and essential morphogenic functions for wall-and lipoteichoic acids in Bacillus subtilis. The EMBO journal, 2009. 28(7): p. 830-842.

60. Carlton, B.C. and B.J. Brown, Gene mutation, in Manual of methods for general bacteriolog, P. Gerhardt, Editor. 1981, American Society for Microbiology: Washington, D.C. p. 222-242

61. Edelstein, A., et al., Computer control of microscopes using microManager. Curr Protoc Mol Biol, 2010. Chapter 14: p. Unit14 20.

62. Yasbin, R.E. and F.E. Young, Transduction in Bacillus subtilis by Bacteriophage SPP1. Journal of Virology, 1974. 14(6): p. 1343.

63. Miller, J.H., A Short Course in Bacterial Genetics: A Laboratory Manual and Handbook for Escherichia Coli and Related Bacteria. 1992: Cold Spring Harbor Laboratory Press.

64. Sambrook, J., Molecular cloning: a laboratory manual, ed. D.W. Russell. 2001, Cold Spring Harbor, N.Y: Cold Spring Harbor Laboratory.

65. Le Breton, Y., N.P. Mohapatra, and W.G. Haldenwang, In vivo random mutagenesis of Bacillus subtilis by use of TnYLB-1, a mariner-based transposon. Applied and Environmental Microbiology, 2006. 72(1): p. 327-333. 


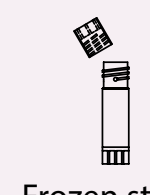

Colony
Frozen stock

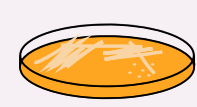

Inoculated fresh media with frozen stock or colony

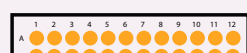
в. c 00009090000 E 000000000000 F 000000000000 F н 000000000000

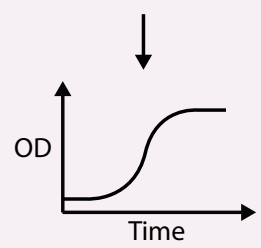

C

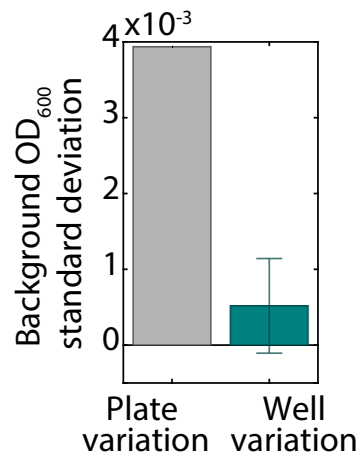

F

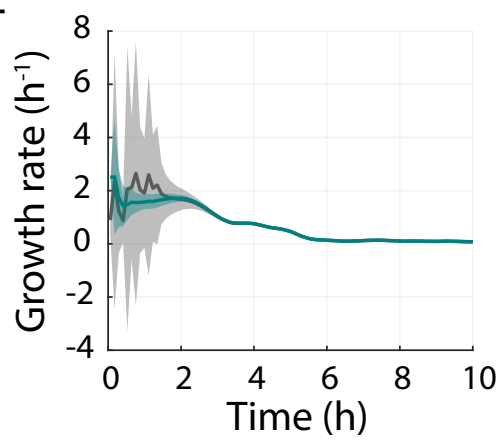

H

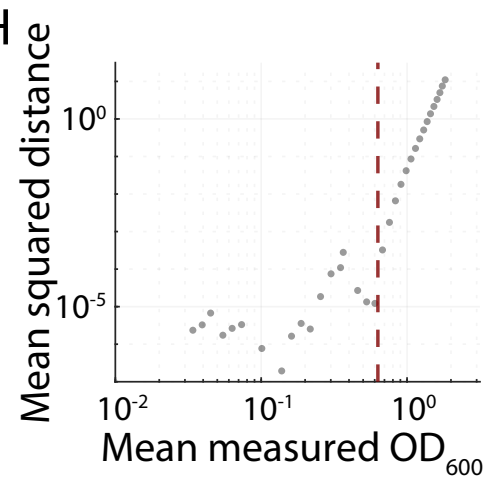
blanking blanking

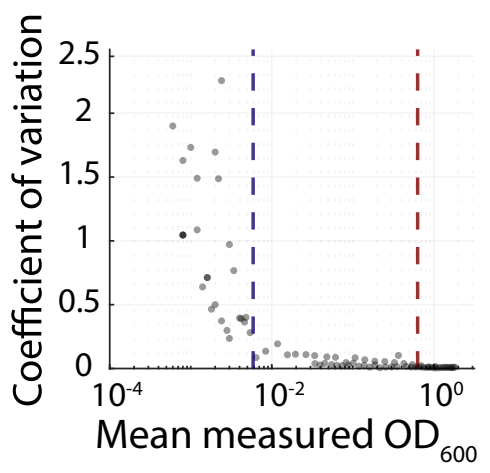

Day 2

$1: 200$ dilution of
overnight into fresh media
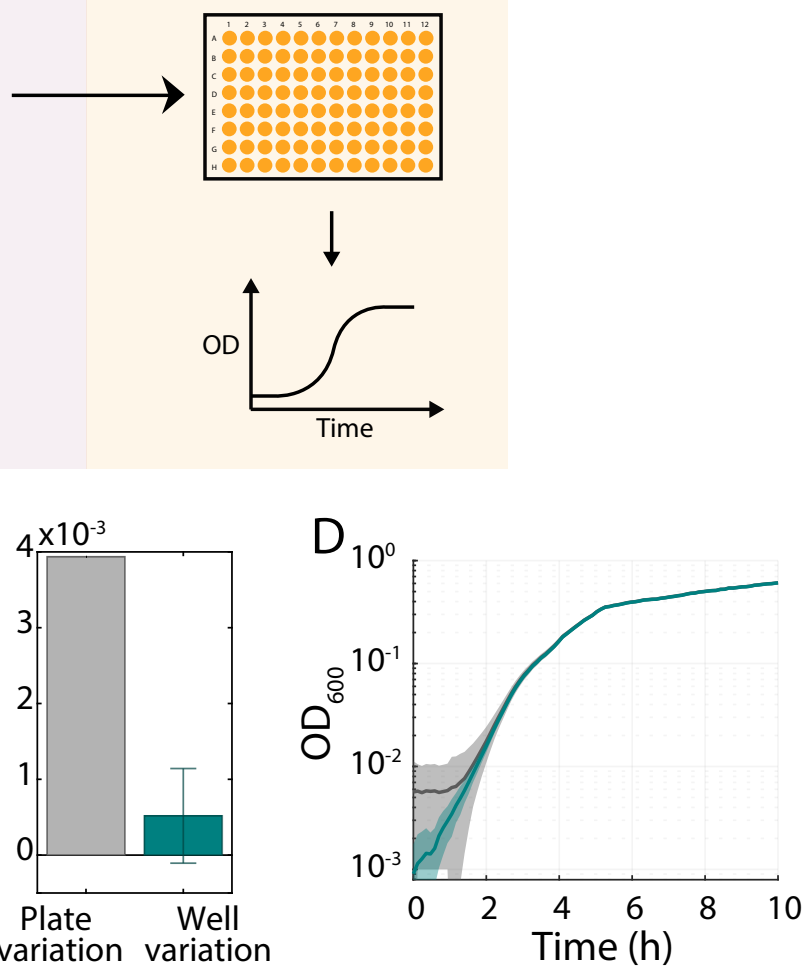

$G$ specific specific

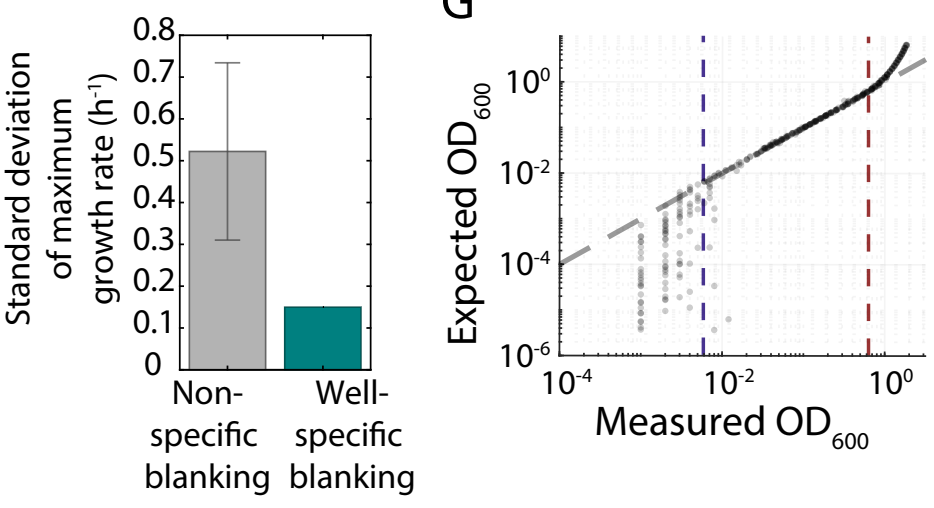


A

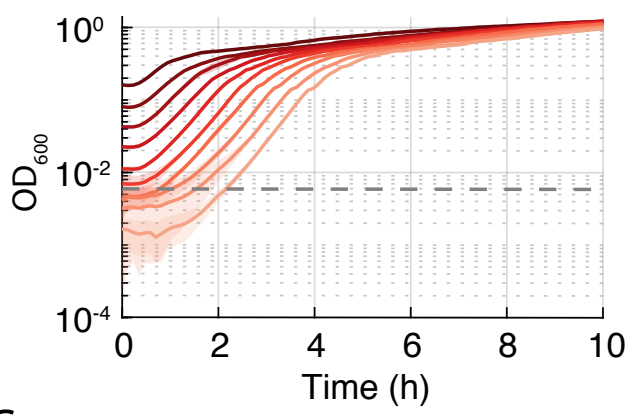

C

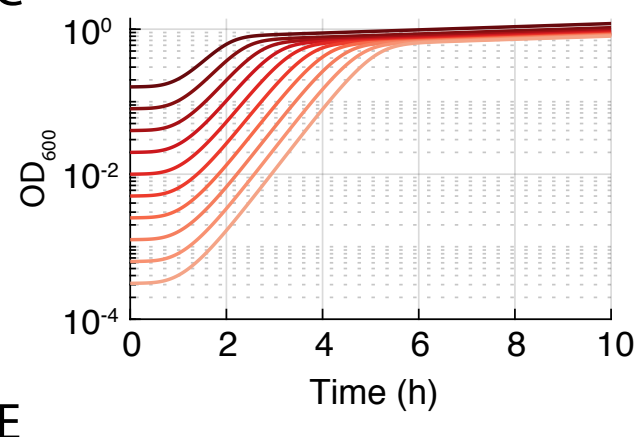

E

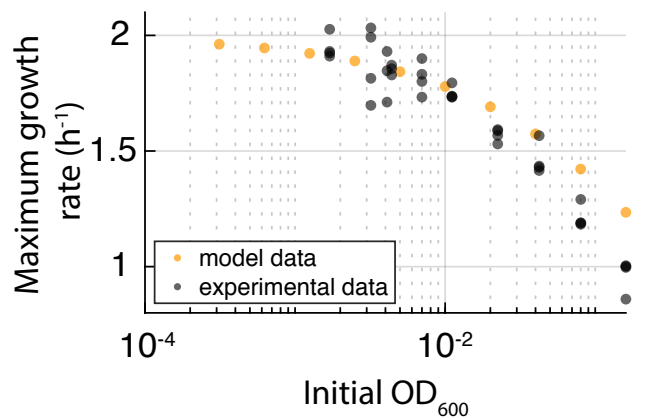

B

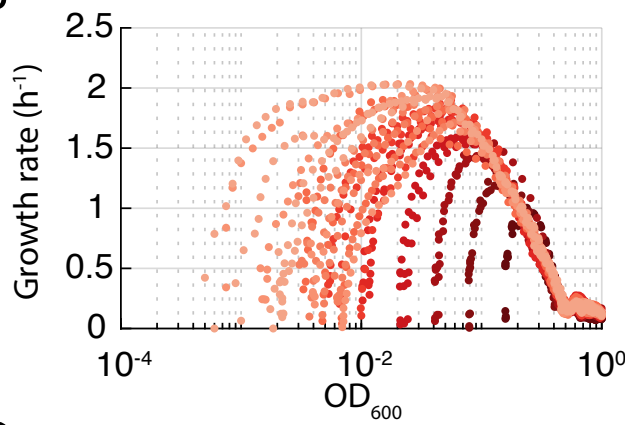

Dilutions

D
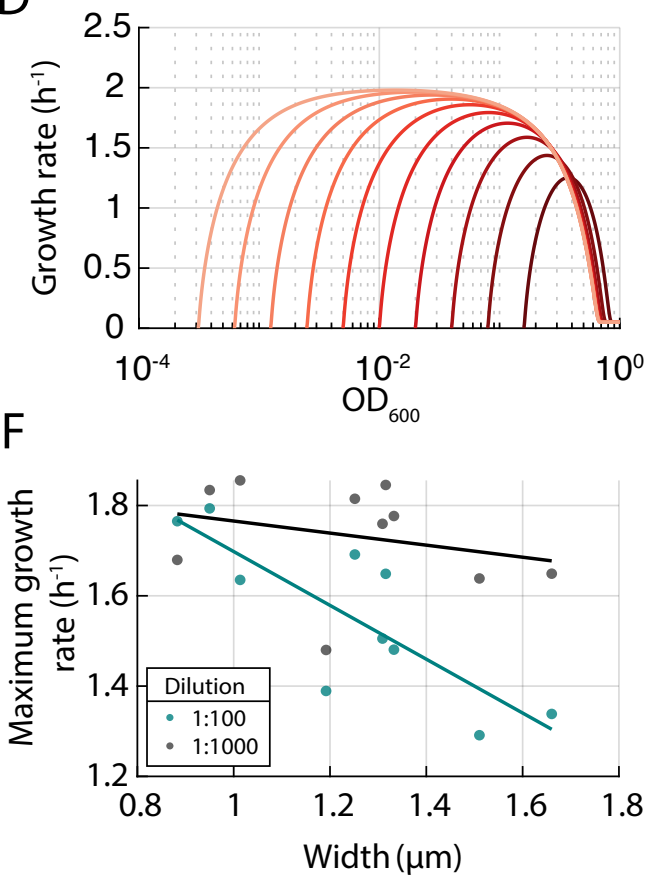


\section{Day 3}

$1 \mu \mathrm{L}$ of frozen stock (with residual glycerol) into DM25 supplemented with $0-10 \% \mathrm{v} / \mathrm{v}$ glycerol

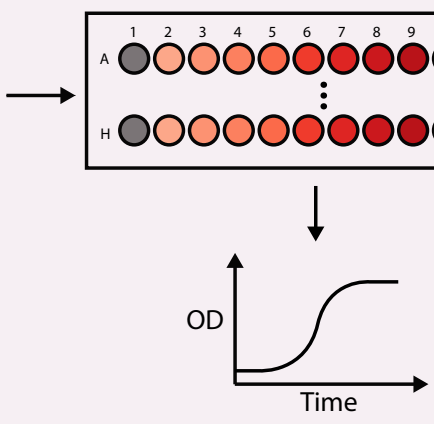

Dilute into DM25 (no added glycerol)

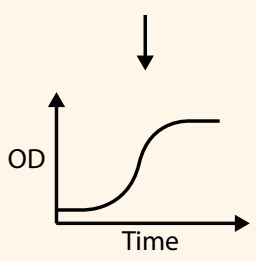

Dilute into DM25 (no added glycerol)

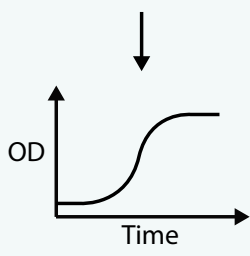

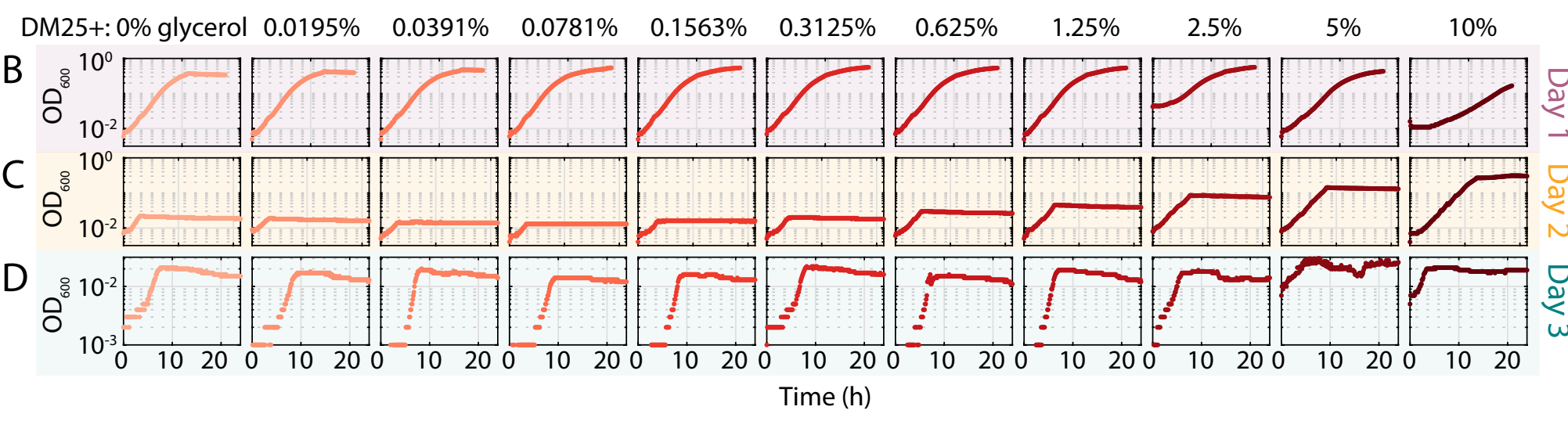

E
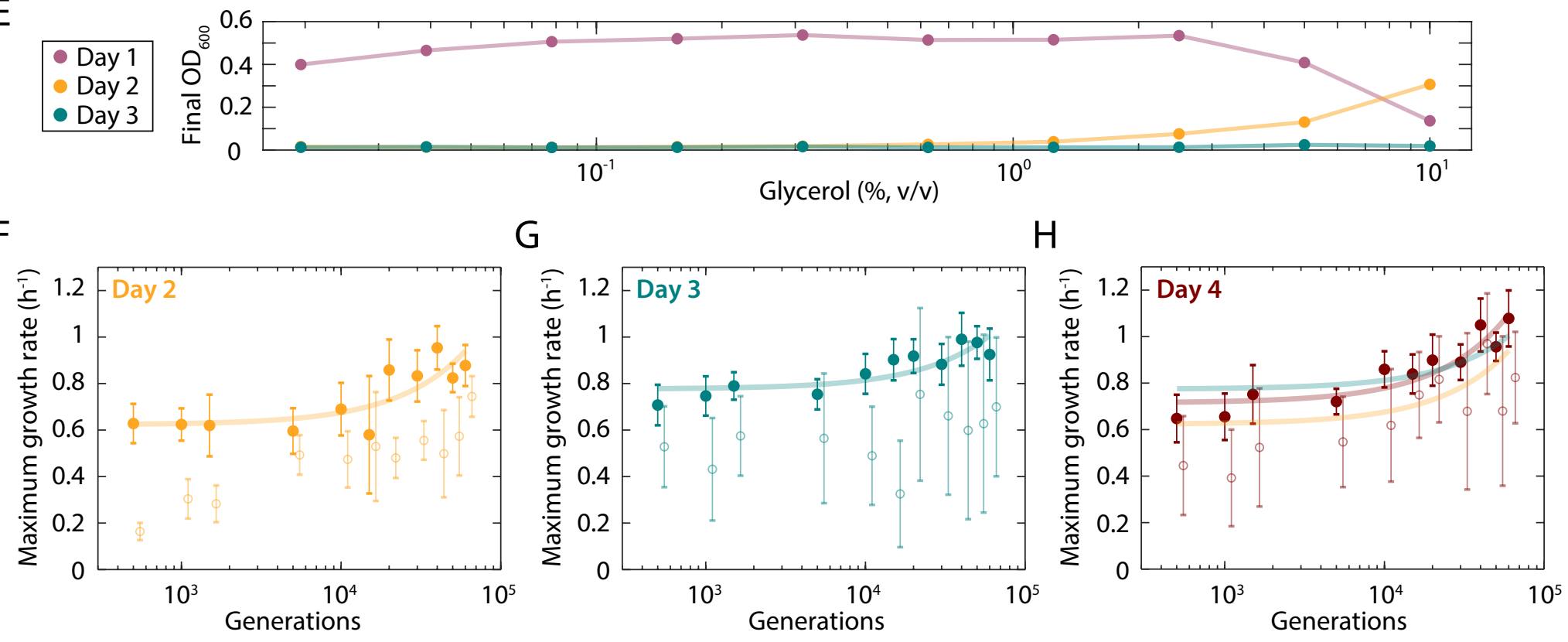
$1 \mu \mathrm{L}$ of frozen stock (with residual glycerol) into LB supplemented with $0-10 \% \mathrm{v} / \mathrm{v}$ glycerol
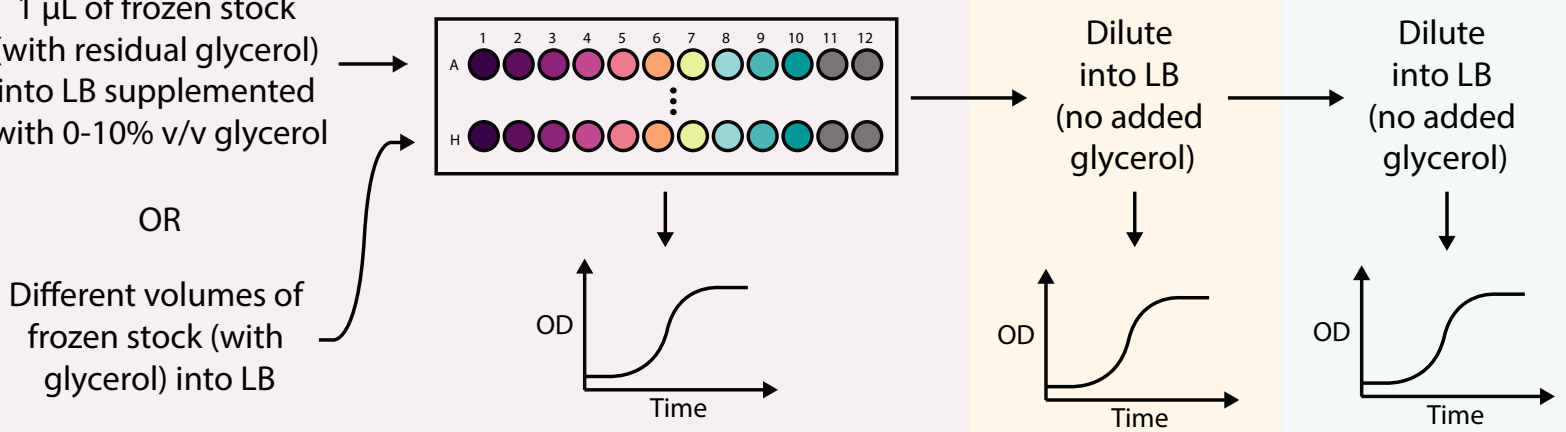

B
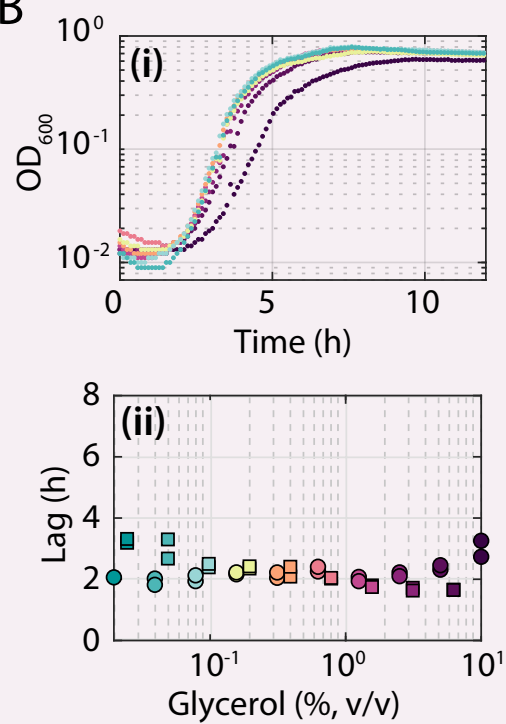
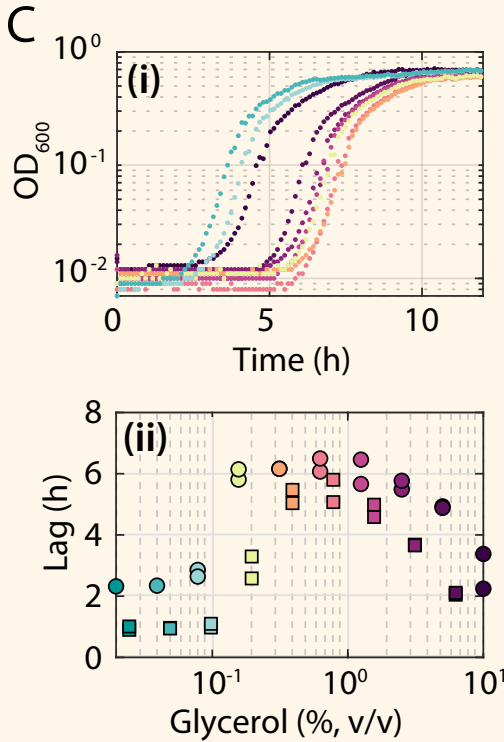
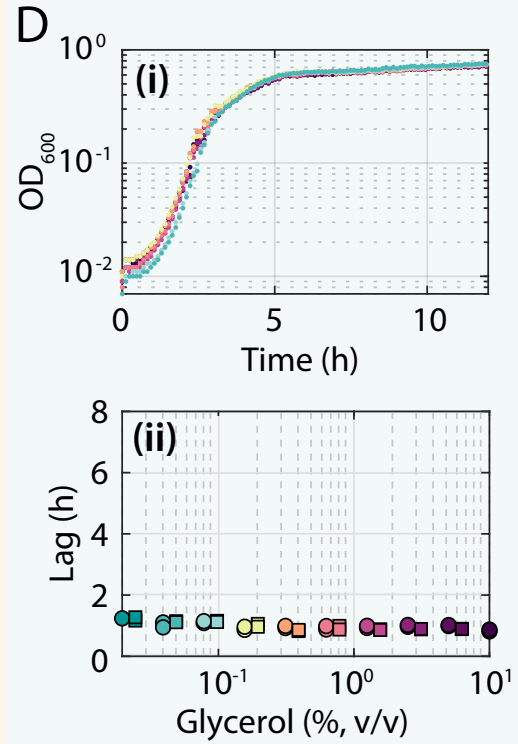

\begin{tabular}{|l|}
\hline \% glycerol \\
\hline$\cdot 10$ \\
$\cdot 5$ \\
-2.5 \\
-1.25 \\
-0.625 \\
-0.3125 \\
0.1563 \\
-0.0781 \\
-0.0391 \\
-0.0196 \\
\hline
\end{tabular}




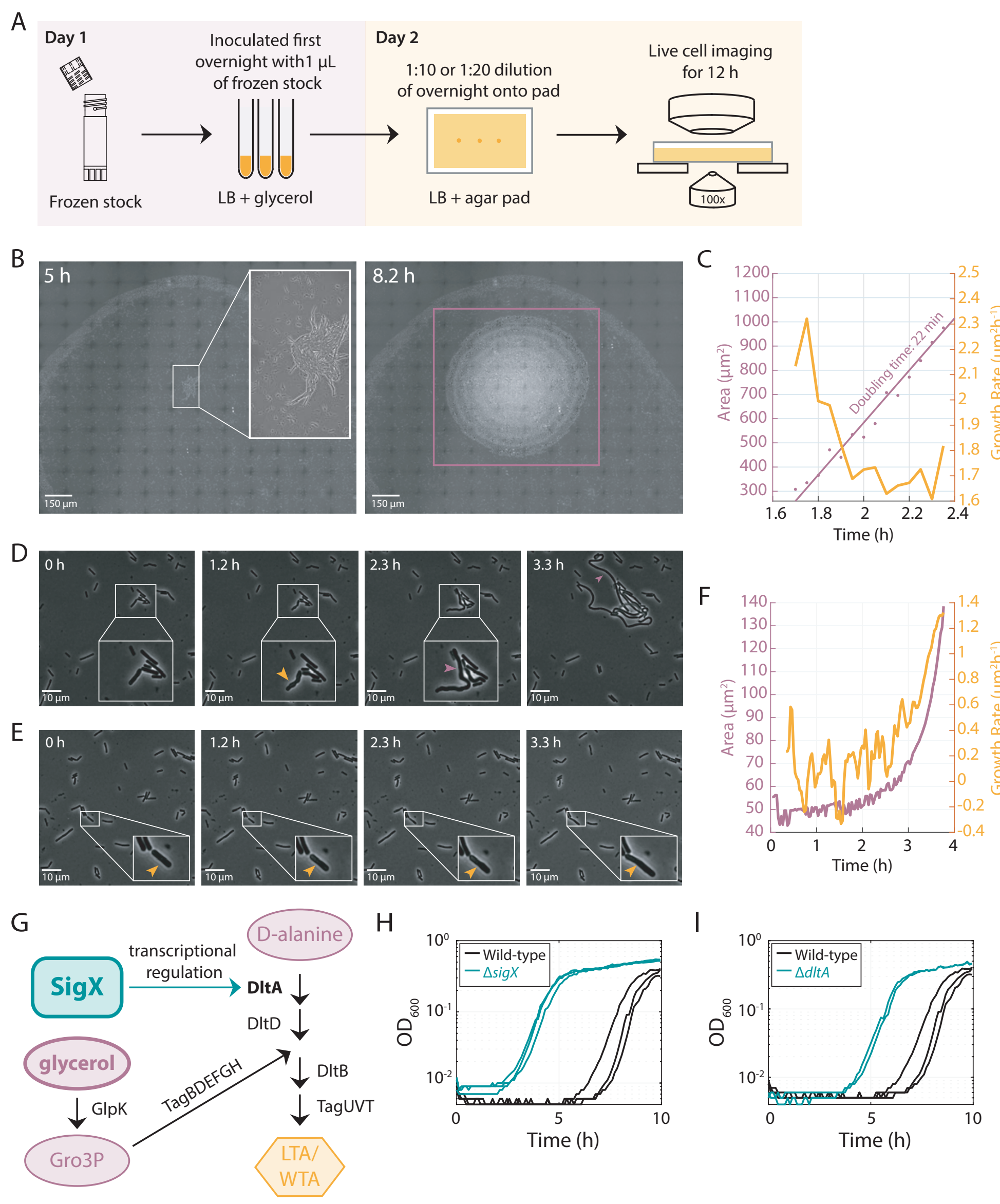

